\title{
Magnetic Resonance Imaging: From Spin Physics to Medical Diagnosis
}

\author{
Pierre-Jean Nacher
}

\begin{abstract}
Two rather similar historical evolutions are evoked, each one originating in fundamental spin studies by physicists, and ending as magnetic resonance imaging (MRI), a set of invaluable tools for clinical diagnosis in the hands of medical doctors. The first one starts with the early work on nuclear magnetic resonance, the founding stone of the usual proton-based MRI, of which the basic principles are described. The second one starts with the optical pumping developments made to study the effects of spin polarization in various fundamental problems. Its unexpected outcome is a unique imaging modality, also based on MRI, for the study of lung physiology and pathologies.
\end{abstract}

\section{Historical introduction}

Magnetic Resonance Imaging (MRI), now widely known for its usefulness as a medical diagnosis tool and for the variety of clear pictures of the body's interior obtained in a harmless and non-invasive manner, had its foundations laid more than 60 years ago in physics experiments designed to measure properties of the nuclear spins of hydrogen atoms. In even earlier experiments, Rabi had shown that an oscillating magnetic field could induce transitions between levels associated to the spin state of various nuclei in an applied static magnetic field [1]. This pioneering work was performed on molecular beams, using a selection and detection method of the nuclear spin state similar to that developed in the 1920's by Stern and Gerlach for their demonstration of spin quantification in silver atoms. The transposition of Rabi's observation of Nuclear Magnetic Resonance (NMR) in a beam of independent molecules to solid and liquid samples was successfully done independently in 1945 by Purcell and by Bloch. ${ }^{1}$ The key feature of both experiments was the observation of the resonance phenomenon through electromagnetic

\footnotetext{
${ }^{1}$ An earlier attempt in Leiden to observe NMR in solids had failed because of exceptionally long relaxation times [9]. Gorter first used the term "nuclear magnetic resonance" in this publication,
} 
detection at the resonance frequency. At the M.I.T., ${ }^{2}$ Purcell, Torrey, and Pound, worked on solid paraffin filling a 1-liter cavity tuned at $30 \mathrm{MHz}$. They observed a $0.4 \%$ change in the rf signal amplitude fed to the cavity due to energy dissipation arising from nuclear spin relaxation of $\mathrm{H}$ atoms [2]. Meanwhile, at Stanford University, Bloch, Hansen, and Packard, performed similar experiments at $7.7 \mathrm{MHz}$ on a 1.5-cc sample of water. They used two orthogonal rf coils: the receive coil detected the re-emission of the resonant rf power absorbed from the transmit coil by the nuclei of the water protons (H atoms) [3, 4]. Although Rabi's work was crucial as the initial demonstration of NMR (he was awarded the Physics Nobel prize in 1944 "for his resonance method for recording the magnetic properties of atomic nuclei"), the conceptual and technical leap achieved by Bloch and Purcell really opened the way to modern NMR and MRI. They were awarded the 1952 Physics Nobel prize "for their development of new methods for nuclear magnetic precision measurements and discoveries in connection therewith". A last essential tool of NMR was found independently by Torrey [5] and Hahn [6], who demonstrated the feasibility of pulsed NMR (initially suggested by Bloch) and observed free Larmor precession. Hahn further used pulsed NMR to generate and observe spin echoes [7].

The next 20 years saw the development of NMR as a powerful investigative tool in many areas of physics and even more so in chemistry. The sensitivity of the nucleus to its electronic environment in a molecule (the "chemical shift") and spinspin interactions were seen at first in the nuclear physics community as annoying features, but the potential of NMR spectroscopy for analytical studies was soon revealed by the discovery of the 3 peaks of ethanol in Purcell's group [8]. Almost none of the early applications of NMR were medical, although a large amount of work was published on relaxation, diffusion, and exchange of water in cells and tissues, even in living human subjects [10] and whole animals [11].

However it was not until 1973 that NMR was used to generate true (2-D) images, when Lauterbur, after his early work on chemical shifts, produced images (of two glass tubes filled with water) reconstructed from a series of 1-D projections obtained using a magnetic field gradient [12]. Soon, MRI benefited from essential developments performed by Mansfield in Nottingham to efficiently generate images, such as slice selection [13] and fast acquisition schemes [14]. These pioneering contributions were jointly rewarded by the 2003 Nobel prize in Medicine "for their discoveries concerning magnetic resonance imaging". Another essential contribution to MRI originated from Zurich, where Ernst applied his Fourier spectroscopy techniques [15] to imaging [16] (Ernst was awarded the Nobel Prize in

attributing the coining of the expression to Rabi. In Kazan, another place where major contributions to NMR were made, Zavoiskii had also failed to reliably detect NMR in 1941, but he discovered electron spin resonance (EPR) in 1944.

${ }^{2}$ Purcell obtained his $\mathrm{PhD}$ degree in 1938 at Havard University, where he returned in 1945, became Professor of Physics in 1949, and performed most of his work. But he spent the war years in the Fundamental Developments Group in the Radiation Laboratory of the M.I.T., where he was associated in particular to Rabi. Purcell's seminal publication on NMR [2] bears the M.I.T. address. 
Chemistry in 1991 "for his contributions to the development of the methodology of high resolution nuclear magnetic resonance spectroscopy").

Research in MRI was pursued only in a few academic laboratories, mostly in the UK, with the first image of a human thorax in 1977, of a head in 1978, until 1980 when Edelstein, in Mallard's team in Aberdeen, obtained the first clinically useful image of a patient [17]. After this date, several companies started to invest in MRI developments and to promote clinical trials. For instance, Toshiba marketed the first commercial MRI scanner in 1983 (with a 0.15 T field), while General Electric, the current leading manufacturer, enrolled several of the MRI pioneers, including Edelstein, and produced the first $1.5 \mathrm{~T}$ clinical system in 1985. With the growing availability and performance of MRI instruments, scientific publications on MRI in medical journals linearly increased in number from 1984, to reach 16000 per year in 2006 [18]. At the same time, MRI exams progressively became a routine diagnosis tool, with now 70 million exams per year and 40 thousand machines in the world.

The rest of this paper is organized as follows. The next section briefly provides the simple notions required to understand the usual NMR dynamics involved in MRI applications. The following section describes the basic principles of imaging, and the influence of elementary physical processes such as relaxation and molecular diffusion on image quality. Finally, the last section is devoted to an unexpected application of decades of developments in the field of polarized noble gas production. This work, that originally aimed at providing tools for various fundamental studies involving spin physics, now also provides an unrivaled tool for MRI of the lung.

\section{Basic physics of NMR}

\subsection{Bloch's equations and NMR dynamics}

The phenomenon of magnetic resonance results from the dynamics of spins in combined static and oscillating magnetic fields. Since, by far, most NMR and MRI applications involve spin $1 / 2$ nuclei, only such spin systems will be considered in the following. NMR indeed also operates for nuclei with higher spin values, but has more complex features. The quantum-mechanical derivation of spin dynamics can be found in many textbooks, and the main results are only recalled here.

In an applied static magnetic field $B_{0}$, the two eigenstates that correspond to the the projections $\pm \hbar / 2$ of the angular momentum on the quantization axis defined by $B_{0}$ have an energy difference:

$$
\Delta E=E_{-}-E_{+}=\hbar \omega_{0}=\hbar \gamma B_{0},
$$

where the transition (Larmor) angular frequency $\omega_{0}$ usually falls in the radiofrequency (rf) domain, and $\gamma$ is the gyromagnetic ratio associated with the spin of the considered nucleus. For protons, the nuclei of $\mathrm{H}$ atoms, $\gamma / 2 \pi=42.58 \mathrm{MHz} / \mathrm{T}$. At thermal equilibrium (for a temperature $T$ ), the system is described by a diagonal 
density matrix with a ratio of populations:

$$
N_{-} / N_{+}=\exp \left(-\hbar \gamma B_{0} / k_{\mathrm{B}} T\right) \text {. }
$$

For a positive gyromagnetic ratio (such as that of protons or ${ }^{13} \mathrm{C}$ for instance, but contrary to the case of the spin $1 / 2$ noble gases ${ }^{3} \mathrm{He}$ and ${ }^{129} \mathrm{Xe}$ that will be considered in section 4), the more populated low-energy state is that with the nuclear spin aligned in the direction of the applied field.

Transitions can be driven between these two eigenstates by a transverse rf field $B_{1}$ (i.e., perpendicular to $B_{0}$ ) rotating at angular frequency $\omega$, and the probability that the system is in the initial state at time $t$ oscillates according to the Rabi formula:

$$
\mathcal{P}(t)=1-\frac{\omega_{1}^{2}}{\omega_{1}^{2}+\left(\omega-\omega_{0}\right)^{2}} \sin ^{2}\left(\frac{t}{2} \sqrt{\omega_{1}^{2}+\left(\omega-\omega_{0}\right)^{2}}\right)
$$

where the amplitude of the rf field, $\omega_{1}=\gamma B_{1}$ (in angular frequency units), and the detuning from resonance, $\omega-\omega_{0}$, determine the characteristic features of the oscillation (see Fig. 1, left) ${ }^{3}$. In the historical Rabi experiment [1], where the fraction of atoms remaining in the initially prepared state is measured after the action of the rf field for a variable time depending on the molecular velocity, the observed resonance curve is indeed described by Eq. 3, simply replacing the oscillating $\sin ^{2}$ term by its time-averaged value, $1 / 2$. An additional effect of the applied resonant rf field is to periodically create coherences (off-diagonal terms) in the density matrix describing the spin $1 / 2$ system. They correspond to transverse components of the angular momentum, which have a time evolution best described in the rotating frame synchronous with the applied rf field (see Fig. 1, right). ${ }^{4}$ An equivalent classical description of the spin dynamics is obtained if one considers a system with an angular momentum $\mathbf{j}$ and a magnetic moment $\mathbf{m}$ such that $\mathbf{m}=\gamma \mathbf{j}$. This vector proportionality ${ }^{5}$ is responsible for the gyroscopic-like response of spins to an applied magnetic field, similar to the dynamics of a spinning top in a gravity field. In a physical sample, the time evolution of the macroscopic local magnetization density $\mathbf{M}$ that results from the magnetic moments of the nuclei behaves in the same way, and thus obeys Bloch's equation [4]. In fact, in most cases, this classical description can be extended to interacting spin systems by simply introducing phenomenological relaxation terms to take into account the stochastic

\footnotetext{
${ }^{3}$ If the transverse field is oscillating, it can be considered as composed of two counter-rotating components, among which only one (with half of the total amplitude) is resonant, whereas the other has no effect.

${ }^{4}$ Since any two-level quantum system is formally equivalent to a spin $1 / 2$ system, such representation is used in many physical problems, even if the levels correspond to electronic states of an atom, connected by an electric dipolar transition. Rydberg atoms in a box are near-ideal examples of such two-level systems [19]. NMR concepts and vocabulary, such as $\pi / 2$ or $\pi$ pulses, are used, and the (optical) Bloch equations rules the evolution of the system.

${ }^{5}$ This non-classical relation does not hold, for instance, for the magnetized needle of a compass. The magnetic moment is a constant locked to the long axis of the needle, but the angular momentum is proportional to the angular frequency of rotation of the needle.
} 

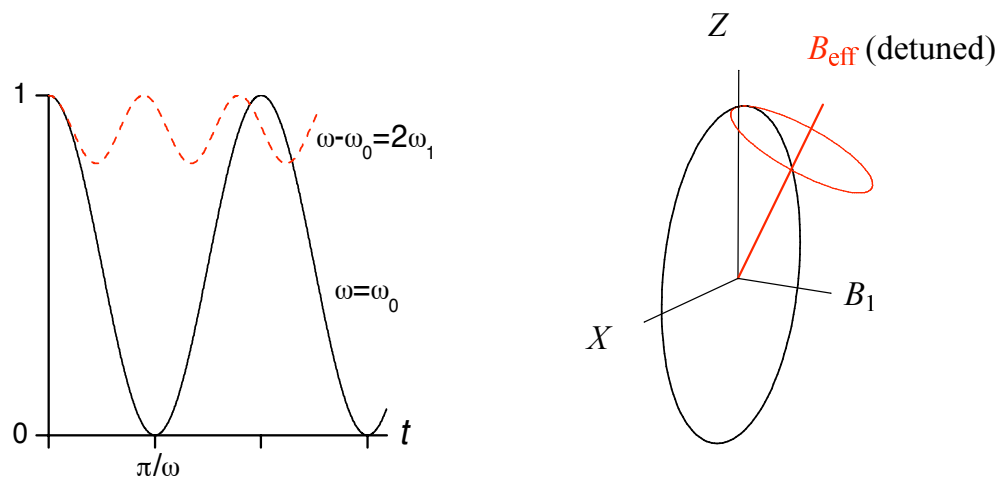

FiguRE 1. Left: time evolution of the probability for a spin $1 / 2$ to be in the initial eigenstate under rf irradiation (Eq. 3). Solid line: resonant $\mathrm{rf}, \omega=\omega_{0}$; dashed line: detuned $\mathrm{rf}, \omega-\omega_{0}=2$ $\omega_{1} ; \omega_{0}$ is the resonance (Larmor) angular frequency, $\omega_{1}$ is the amplitude of the rotating rf component in angular frequency units. Right: The corresponding trajectories of the classical momentum associated with this spin are circles on the so-called Bloch unity sphere. The longitudinal $(Z)$ component is the Rabi oscillation probability plotted on the left, and the projection in the transverse $(X Y)$ plane or represents the off-diagonal elements in the spin density matrix ( $Y$ is the direction of the rf field).

effect of dipolar spin interactions. ${ }^{6}$ This leads to the usual Bloch's equation in its complete form:

$$
\frac{d \mathbf{M}}{d t}=\gamma \mathbf{M} \times \mathbf{B}_{\text {eff }}-[R] \mathbf{M}-D \boldsymbol{\Delta} \mathbf{M} .
$$

It is most conveniently written in the rotating frame synchronous with the rf field. The effective field in the first term of Eq. 4 is then given by:

$$
\mathbf{B}_{\text {eff }}=-\omega_{1} \hat{x} / \gamma-\left(\omega-\omega_{0}\right) \hat{z} / \gamma .
$$

When no rf field is applied, a frame rotating at the local Larmor frequency is most convenient (then $\mathbf{B}_{\text {eff }}=0$ ), but a frame rotating at any frequency $\omega$, e.g. the average Larmor frequency over the extended physical sample, can also advantageously be used (then $\mathbf{B}_{\text {eff }}$ is given by Eq. 5 with $\omega_{1}=0$ ). The second term in Eq. 4 is a relaxation term characterized by two different rates: one for the longitudinal component $M_{z}$ of $\mathbf{M}$, noted $1 / T_{1}$, and one for the transverse components, noted

\footnotetext{
${ }^{6} \mathrm{~A}$ full quantum treatment of the spin dynamics is required only in rare particular cases, for instance when short-range quantum correlations between interacting spins of nuclei in a molecule play a key role. Such unusual situations will not be considered here, and the classical description will always be used. A discussion regarding the necessity of using a quantum formalism can be found in [23] and references therein.
} 
$1 / T_{2}$. The last term in Eq. 4 describes the irreversible effect of atomic diffusion, that depends on the spin diffusion coefficient $D$.

In cw NMR with weak rf fields, relaxation phenomena play an important role to determine the response of the spin system to irradiation, and hence deeply influence the observed spectra. In contrast, in pulsed NMR, as performed for MR imaging (see section 3), the rf field is usually so intense that relaxation and diffusion phenomena have no significant influence during the pulse. Hence the right hand side of Bloch's equation (Eq. 4) is reduced to its first term, and $\mathbf{M}$ simply undergoes a rotation around the direction of the effective field. At resonance, any tip angle with respect to the initially longitudinal orientation of $\mathbf{M}$ can be achieved using an appropriately timed tipping rf pulse (see Fig. 1, right). Finally, in the absence of applied rf (e.g., during the free evolution following a tipping pulse), the complete Bloch's equation shows that a uniform magnetization in a uniform field $B_{0}$ simply precesses at Larmor frequency around the field axis, with a transverse component that decays exponentially (with a rate $1 / T_{2}$ ) and a longitudinal component that exponentially recovers its equilibrium value, determined by Eq. 2 (with a rate $1 / T_{1}$ ). If the magnetic field $B_{0}$ is not uniform (as is inevitably the case in an experiment), the transverse part $\mathbf{M}_{\perp}$ of $\mathbf{M}$ progressively acquires a non-uniform phase, and magnetization currents induce an additional decay of $\mathbf{M}_{\perp}$ according to the third term in Bloch's equation.

\subsection{Signal amplitude considerations}

In the historical Rabi experiment, the resonance was observed by monitoring the flux of molecules in a weak but fully polarized beam. Similarly, optical detection of magnetic resonance in optically pumped atomic vapors, proposed by Kastler ${ }^{7}$ [21] and demonstrated by Brossel and Cagnac [22], was a very sensitive method to observe magnetic resonance phenomena in very dilute systems. The common feature of these approaches is that the system's polarization is complete or very high, and that the detection of atoms or of visible photons, with energies in the eV range, can provide excellent signal-to-noise ratios (SNRs) even with a modest number of detected events. In contrast, standard NMR with radioelectric detection of the resonance, as pioneered by Purcell and Bloch in their seminal work, relies on the detection of (magnetic) rf oscillations involving much lower energies (NMR frequencies are in the $\mathrm{MHz}$ range, $10^{-9}$ times lower than optical frequencies). Moreover it is performed in weakly polarized samples (from Eq. 2, equilibrium polarization is of order $10^{-5}$ at room temperature for $B_{0}=1 \mathrm{~T}$ ). Altogether, a dramatic loss of sensitivity (of 14 orders of magnitude) is expected to result from these combined reductions in signal amplitude. Fortunately, this is compensated by a huge increase in density from molecular beams or dilute atomic vapors to liquid or solid samples.

\footnotetext{
${ }^{7}$ A. Kastler was awarded the 1966 Nobel prize in physics "for the discovery and development of optical methods for studying Hertzian resonances in atoms". The principle of optical pumping, and its application to the obtention of nuclear polarization in noble gases, will be described in section 4 .
} 
Indeed, such a discussion on signal amplitudes only is rather crude, and more careful SNR arguments must be considered. NMR signal during free precession is usually picked up by a coil that converts the oscillating flux of precessing magnetization into a recorded electromotive force (emf). For given sample and coil geometries (their effect is not discussed here), the emf simply scales with $B_{0}^{2}$ because the equilibrium magnetization is proportional to $B_{0}$, and because Faraday's law introduces a time derivative, hence a factor $\omega_{0}$. Estimating the field dependence of noise is more delicate, and implies making assumptions on its physical origin. Disregarding instrumental noise arising from rf interference (efficiently suppressed when operating in a Faraday cage or shielded room) or generated by amplifiers and recording electronics, unavoidable noise originates from the coil and from the sample. This thermal (Johnson-Nyquist) noise results from thermal agitation of charge carriers, in the coil and in the sample. For the latter, Faraday's law introduces the same factor $\omega_{0}$ into the induced emf as in does for the signal, so whenever noise mostly originates from the sample, SNR increases only linearly with $B_{0}$. In contrast, whenever noise mostly originates from the coil, it can be argued that SNR increases more rapidly with the operating field, scaling as $B_{0}^{7 / 4}[24]$. For a well-designed coil and for medical MRI applications, the sample (i.e., the patient's tissues) is the dominating source of noise for sample sizes exceeding a few centimeters. Conversely, coil noise dominates for non-conducting samples, for MRI of small animals or for MR microscopy using small-sized surface coils. In this case, it can be efficiently reduced using cold probes, or even superconducting coils [25]. The thermal noise spectral density, that is given by $\sqrt{4 k_{B} T R}$ (across a resistor $R$ at temperature $T$ ), is thus decreased through the reduction both of $R$ and $T$. More exotic options are being explored to further improve SNR in MRI, such as the use of SQUID-based devices [26] or of optical magnetometers [27] at very low field, or of force detection in magnetic resonance force microscopy for sub-micron resolution MRI [28].

\section{Principles of MRI}

\subsection{Effect of an applied field gradient: 1-D imaging}

When NMR is performed in a position-dependent magnetic field $B_{0}(\mathbf{r})$, the Larmor precession frequency correspondingly depends on position $\mathbf{r}$ in the sample. An important practical case is met when a uniform field gradient $G \hat{\mathbf{k}}$ is applied:

$$
B_{0}(\mathbf{r})=B_{0}(0)+G \hat{\mathbf{k}} \cdot \mathbf{r} .
$$

The precession frequency only depends on position along the direction $\hat{\mathbf{k}}$ of the gradient, and resonance synchronously occurs in any plane perpendicular to $\hat{\mathbf{k}}$. If cw NMR is performed, signal at a given frequency is induced by atoms the vicinity of one such plane (there is in fact a limit to the accuracy of the localization resulting from the NMR intrinsic line width for $G=0)$. If the NMR signal is received using a coil with uniform coupling to all parts of the sample (e.g., using a 
long solenoidal coil), NMR spectra recorded when sweeping the field or the frequency represent 1-D projections of the magnetization density in the sample. The frequency scale is set by the amplitude $G$ of the gradient, and the projection is actually convolved with the Lorentzian line shape corresponding to the relaxation rate $1 / T_{2}$, which limits the achievable resolution along the direction of the gradient to a scale $\delta r=1 / \gamma G T_{2}$. This is illustrated in Fig. 2 for the case of a spherical sample with uniform magnetization density. In the remainder of this section, we shall assume that large values of $G$ are used $\left(\gamma G \rho T_{2} \gg 1\right)$, and moreover that diffusion effects can be neglected.

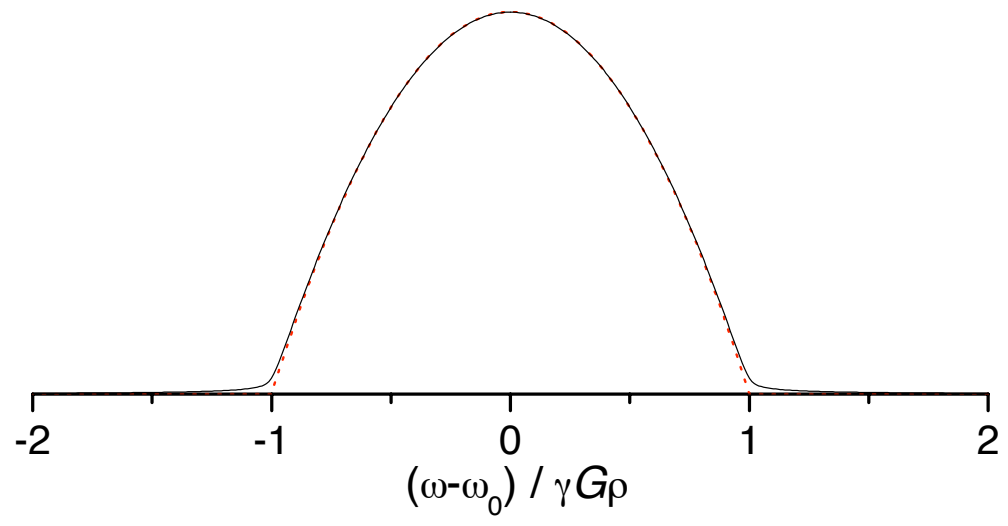

FIgURE 2. Computed cw NMR spectra for a uniform spherical sample of radius $\rho$ in a uniform gradient of amplitude $G$. Without relaxation (dashed line), the spectrum is the positive part of the parabola 1- $\left(\omega-\omega_{0}\right)^{2} /(\gamma G \rho)^{2}$, directly mapping the sections of the sphere. With some amount of relaxation in Bloch's equation (but neglecting diffusion effects), sharp edges are smoothed out (solid line: $\left.\gamma G \rho T_{2}=0.01\right)$.

Pulsed NMR is actually preferred to the very time-consuming cw NMR for imaging applications. Following a tipping pulse, the free-precessing magnetization induces an emf in the coil:

$$
e(t) \propto \int_{\text {sample }} M(\hat{\mathbf{k}} \cdot \mathbf{r}) \cos \left[\gamma B_{0}(\mathbf{r}) t+\varphi\right] e^{-t / T_{2}}
$$

where the local value of the field (Eq. 7) sets the local Larmor frequency, and $\varphi$ is a phase term depending on the tipping pulse and on the coil position. The actually recorded NMR signal is obtained by beating the high-frequency voltage $e(t)$ with a reference voltage at a fixed frequency $\omega_{\text {ref }}$, e.g. $\gamma B_{0}(0)$. The resulting in-phase and quadrature voltages are the components of a complex signal $S(t)$ :

$$
S(t)=\int_{\text {sample }} M(\hat{\mathbf{k}} \cdot \mathbf{r}) \exp [i \gamma t G \hat{\mathbf{k}} \cdot \mathbf{r}] e^{-t / T_{2}}
$$


that represents the sum of contributions from all parts of the sample in the frame rotating at the reference frequency $\omega_{\text {ref }}$. This complex signal keeps track of the sense of rotation in the rotating frame, and thus discriminates between frequencies higher and lower than $\omega_{\text {ref }}$. The time evolution of $S$ is illustrated in Fig. 3 for the same spherical sample as in Fig. 2. A Fourier transform (FT) allows one to switch

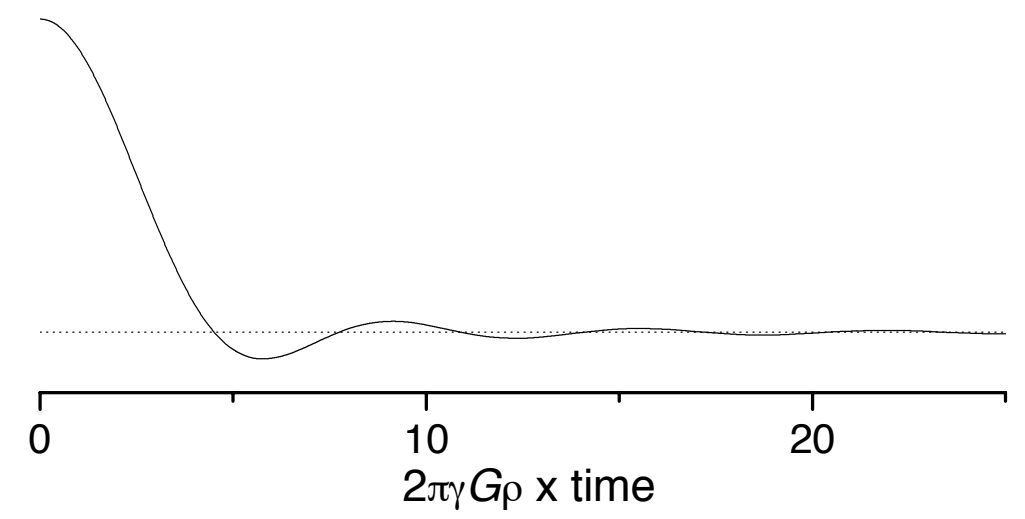

Figure 3. Computed NMR signal (Eq. 8) for a uniform spherical sample of radius $\rho$ in a uniform gradient of amplitude $G$ (neglecting relaxation and diffusion effects). The signal has a constant phase, and the quadrature (dotted line) remains null at all times.

from the time domain to the frequency domain, and to obtain from this signal the spectrum that would be recorded in a cw experiment. Although the knowledge of $S$ only at positive times is in principle sufficient to derive the spectrum, hence the 1-D projection image of the sample (computing the FT of the function defined from the signal by setting $S(-t)=S^{*}(t)$ ), gradient-echo or spin-echo techniques are often used to acquire more information in the time domain.

The fast decay of the signal, with a time scale of order $1 / \gamma G \rho$ for a sample of size $\rho$, essentially results from the phase decoherence of the local magnetization. Since the local magnetization has hardly decayed under the action of relaxation, the phases can be refocused, and a so-called gradient echo can be formed with a large value of the signal recovered at a finite time after the tipping pulse. Phase refocusing can result from an inversion of the gradient at time $\tau$, after which the signal is given by:

$$
S(t>\tau)=\int_{\text {sample }} M(\hat{\mathbf{k}} \cdot \mathbf{r}) \exp [i \gamma(2 \tau-t) G \hat{\mathbf{k}} \cdot \mathbf{r}] e^{-t / T_{2}}
$$

The initially fast precessing spins become slow precessing ones after the gradient sign change, and all phases are back to their initial values at time $2 \tau$, i.e. the magnetization is uniform again. Following this, evolution proceeds as it does just following a tipping pulse (see Fig. 3). A more general time variation of $G$ can 
be used, with different amplitudes before and after the time $\tau$. $G$ can also be periodically reversed at times $\tau, 3 \tau, 5 \tau, \ldots$ : an echo is formed whenever the time integral of $G(t)$ vanishes and an echo train is obtained.

Another method to obtain an echo consists in leaving the gradient unchanged, but in inverting the sign of all phases at time $\tau$ using a $\pi$ rotation around any direction in the transverse plane. The phase advance of the "fast" spins suddenly becomes a phase lag, and rephasing occurs again at time $2 \tau$. These so-called spin echoes can also be periodically refocused by repeating the $\mathrm{rf}$ pulse inducing the $\pi$ rotation.

Signals obtained using a spin echo or a gradient echo technique are similar, but experimental constraints or imperfections may support the choice of either technique. The main drawback of the gradient echo technique is that gradients can only be approximately reversed. Indeed, the applied gradient that results from current fed to a dedicated set of coils (the gradient coils) can be reversed. But the unavoidable static field map imperfections (that are usually not a uniform gradient) induce a progressive loss of phase coherence that is unaffected by the inversion of $G$ at time $\tau$. Altogether, a gradient echo can be observed only during the the experimental coherence time of the signal, $T_{2}^{*}$, that is often shorter than the transverse relaxation time $T_{2}$. This limitation is removed by the use of a spin echo technique, since the dephasing due to all field variations (both $G$ and the imperfections of $B_{0}$ ) is exactly refocused. However frequently firing intense rf pulses to induce $\pi$ rotations requires a fair amount of power, which may in some cases pose safety problems due to tissue heating.

The time variation of the signal is sampled, with a sampling frequency $f_{\mathrm{s}}$, during a time window of duration $T_{\text {obs }}$ that is usually centered ${ }^{8}$ on the echo time $2 \tau$. The spectrum obtained by FT from these data has a frequency resolution $1 / T_{\text {obs }}$ and extends over a frequency range $\pm f_{\mathrm{s}} / 2$. For the 1 -D projection of the sample, the frequency resolution corresponds to a spatial resolution of $1 / \gamma G T_{\text {obs }}$. Of course this is only a maximal resolution, that is reduced due to transverse relaxation time $\left(T_{2}\right.$, see Fig. 2, or $\left.T_{2}^{*}\right)$, and also due to SNR limitations. The frequency range implies a so-called field of view (FOV) of $f_{\mathrm{s}} / \gamma G$. The FOV must be larger than the sample size to avoid folding artifacts resulting from the undersampling of high frequencies.

\subsection{2-D and 3-D imaging methods}

Various strategies can be used to obtain information on distribution of magnetization in a sample, based on extensions of the 1-D projection imaging method.

\footnotetext{
${ }^{8}$ This is not in fact mandatory. Using symmetry properties of echoes that result from the fact that the magnetization phase is uniform at $t=0$, half of the echo is sufficient to provide the information needed to retrieve spectral data. It is only important to include the echo time $2 \tau$ in the recording. It is technically difficult in simple pulsed NMR to have the full decay from $t=0$ due to the delay for recovery of saturated detection electronics following the rf tipping pulse. A symmetric echo is usually recorded to increase SNR, but asymmetric echoes are used is ultra-fast acquisition schemes.
} 
A widely used technique is the selective rf excitation of only part of the sample, or slice selection. If a rf tipping pulse is applied in the presence of a gradient $G \hat{\mathbf{k}}$, it is non-resonant, and has thus no tipping action, over all of the sample but in the plane defined by $\hat{\mathbf{k}} \cdot \mathbf{r}=\left(\omega-\omega_{0}\right) / \gamma G$. The time duration of the rf pulse determines the thickness of the slice in which significant tipping occurs, and the time envelope of its rf amplitude sets the variation of the tip angle with position. For small tip angles, these are indeed linked by FT relations, and uniform tipping in a slice of given thickness is obtained using a sinc-shaped rf pulse. More complicated pulse shaping [29] is required at large tip angle because of the nonlinear variation of the angle with rf amplitude and detuning (see Fig. 1). 3-D imaging of the sample can be performed combining slice selection, and 2-D imaging in each selectively excited plane.

The standard 2-D cartesian imaging technique consists in acquiring a series of gradient echoes obtained in an applied (so-called readout) gradient $\mathbf{G}_{\mathrm{r}}$, using a variable (so-called phase-encoding) gradient $\mathbf{G}_{\mathrm{e}}$ applied along a perpendicular direction before the echo is obtained. For simplicity, we assume here that $\mathbf{G}_{\mathrm{r}}$ is oriented along the $x$-axis, and $\mathbf{G}_{\mathrm{e}}$ along the $y$-axis, but all orientations can be freely chosen. The recorded signal is thus modified with respect to Eq. 9 and reads:

$$
S(t>\tau)=\int_{\text {sample }} M(x, y) \exp \left[i \gamma \tau_{\mathrm{e}} G_{\mathrm{e}} y\right] \exp \left[i \gamma(2 \tau-t) G_{\mathrm{r}} x\right] e^{-t / T_{2}} .
$$

For each value of the phase-encoding parameter $\tau_{\mathrm{e}} G_{\mathrm{e}}$, a Fourier component of the spatial modulation of the magnetization in the sample along the $y$-axis is probed (see Fig. 4, left). With $N_{\mathrm{e}}$ data acquisitions performed for evenly-spaced gradients amplitudes between $-G_{\max }$ and $G_{\max }$, the so-called k-space (here a 2-D array of data filled recorded time-varying signals) contains sufficient information to compute a 2-D image by (discrete) inverse FT of the data. The FOV in the physical direction of the readout gradient is given by the formula of the previous section 3.1, while in the direction of the encoding gradient the FOV given by $N_{\mathrm{e}} / 2 \gamma \tau_{\mathrm{e}} G_{\max }$.

The original radial projection method introduced by Lauterbur [12] can be used with a series of gradient-echo acquisition of data, for successive gradient orientations $\hat{\mathbf{k}}$ sampling a plane (see Fig. 4). Image reconstruction can be performed using back-projection algorithms, or by considering that the acquired data provide enough information on the 2-D FT of the image (the k-space) to perform an inverse FT after regridding and interpolation. Radial acquisition is often preferred when fast movements are recorded: at the time of each echo, the center of the Fourier space is re-sampled, which eliminates movement artifacts that deeply affect other imaging methods, and allows high time resolution using sliding window methods.

There are indeed other strategies to acquire k-space data, e.g. following different trajectories (spiral or interleaved spiral trajectories), or performing only partial (e.g. half-plane) acquisition [30]. Current advances in fast MRI involve both hardware and software developments. Parallel data acquisition using coil arrays to directly provide spatial information allows one to undersample the k-space [31, 32]. 

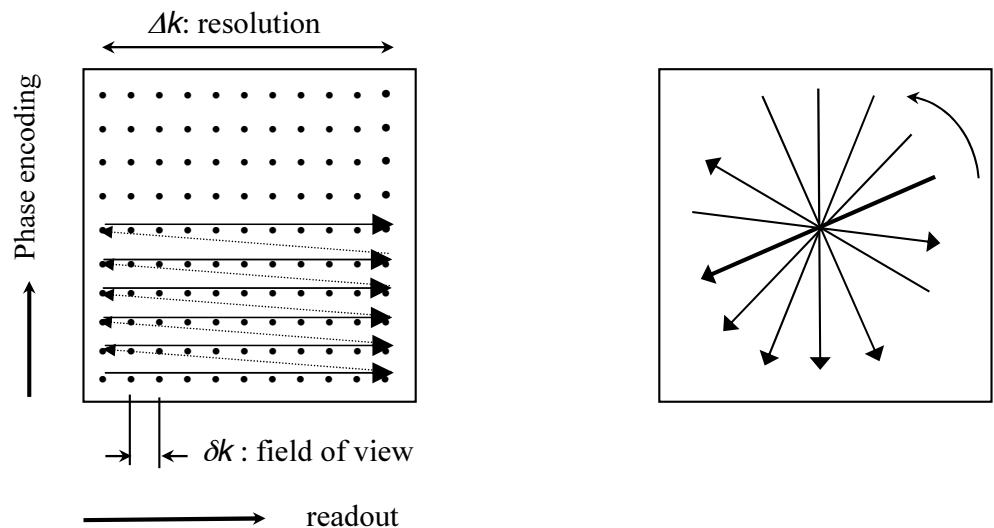

FIgURE 4. Examples of trajectories for 2-D k-space sampling. Left: cartesian mapping of the plane is obtained by a series of data acquisitions with steps of phase encoding in the Y direction. Right: radial mapping is obtained by projections onto different successive directions.

Sparse sampling of the k-space exploiting the spatial or temporal redundancy that usually allows image compression can also provide high-quality images [33]. All these techniques aim at accelerating data acquisition in order to reduce movement artifacts or to capture relevant anatomical motion (e.g. in cardiac imaging). Another motivation is that imaging sessions can take up to several tens of minutes for each patient, because of the large number of data required for high-resolution multi-slice or 3-D images, and due to the necessary signal averaging to obtain high SNR. Reducing the examination time can reduce patient discomfort and allows more cost-efficient use of the clinical MRI systems.

\subsection{Contrast mechanisms}

For all the above imaging strategies, the collected signal and the resulting image reflect the local magnetization density in the sample. This scales with the density of $\mathrm{H}$ atoms, which is high both in water and lipids of all tissues. This provides anatomical images that are usually poorly contrasted and of little clinical use. Fortunately, various physical effects can be put to use to provide enhanced contrast between different organs with similar proton density, or reveal differences between normal and pathological regions.

$T_{1}$-weighting is obtained in imaging sequences where the repetition period $T_{\mathrm{R}}$ of NMR pulses is of the order of the longitudinal relaxation time. In steadystate, the local magnetization is reduced from its thermal equilibrium value $M_{\text {eq }}$ according to:

$$
M=M_{\mathrm{eq}} \frac{1-\exp \left(-T_{\mathrm{R}} / T_{1}\right)}{1-\cos \alpha \exp \left(-T_{\mathrm{R}} / T_{1}\right)},
$$


where $\alpha$ is the tip angle of the rf pulse. For an appropriate choice of $\alpha$ and $T_{\mathrm{R}}$, parts of the sample with short $T_{1}$ will provide a stronger signal and will thus appear more intense in images than parts with a long $T_{1}$, where magnetization does not recover much between tipping pulses. In normal tissues, fat (lipids) has a shorter $T_{1}$ than water and thus appears white in $T_{1}$-weighted images (e.g., cerebral white matter appears so since it contains more lipids than grey matter). Paramagnetic contrast agents can be injected to locally induce a reduction of $T_{1}$ and thus increase signal intensity wherever blood perfusion is present.

$T_{2}$-weighting is obtained in imaging sequences where the signal acquisition time is delayed long enough after the tipping pulse (until the echo time $T_{\mathrm{e}}$ ) for attenuation of the transverse magnetization ( $T_{2}$ in Eq. 9 or 10) to be significant. $T_{2}$-weighted images appear reversed compared to $T_{1}$-weighted images, with bright water-containing regions and weaker signal from lipids. Both $T_{1}$ - and $T_{2}$-weighted images are acquired for most medical examinations. Together, they provide the trained radiologist with a lot of morphological and functional information, since for instance flowing blood, haematomas, and various types of tumors differently affect signal intensity in MR images.

Diffusion is the last physical effect (neglected so far) that affects spin dynamics. Diffusion-weighted images are obtained by adding a bipolar gradient pulse following the tipping rf pulse before the rest of the usual imaging sequence. This first imprints a helix-like phase pattern along the direction of this added diffusionsensitizing gradient, then unwinds it. The net result is that the transverse amplitude is reduced by a factor $\exp \left(-D \gamma^{2} G^{2} \tau_{\mathrm{d}}^{3}\right)$, where $D$ is the diffusion coefficient, $G$ the amplitude and $\tau_{\mathrm{d}}$ a time scale associated with the sensitizing gradient. In the human brain, water diffusion is impeded by natural barriers (cell membranes, myelin sheaths, ...) and a reduced apparent diffusion coefficient (ADC) is measured instead of $D$. ADC imaging of the brain is routinely performed in case of stroke (ischemic or hemmoragic). ADC mapping can also be performed as a function of the direction of the sensitizing gradient. The resulting diffusion-tensor images allow for the determination of directionality as well as the magnitude of water diffusion. This kind of MR imaging enables to visualize white matter fibers in the brain and can for instance map subtle changes in the white matter associated with diseases such as multiple sclerosis or epilepsy.

\section{Lung MRI with polarized noble gases}

In spite of its high abundance in tissues, $\mathrm{H}$ is not the only element that can be detected and imaged by NMR. Non-proton MRI, although it is not part of routine clinical exams, allows for instance useful investigation of the human heart when tuned to the frequencies of ${ }^{31} \mathrm{P}$ or ${ }^{23} \mathrm{Na}$, which are both naturally abundant isotopes with non-zero spin. The low abundance of ${ }^{13} \mathrm{C}(1 \%)$ prevented carbon-based MRI until ex-vivo pre-polarization of its nuclei in selected bio-compatible molecules was used to provide dramatic signal enhancement [34, 35]. 
In addition to these nuclei, that are naturally present in biological tissues, the spin $1 / 2$ noble gases ${ }^{3} \mathrm{He}$ and ${ }^{129} \mathrm{Xe}$ can be used to image the lung airways. This was first demonstrated in 1994 by a Princeton-Stony Brook collaboration using polarized xenon in excised mouse lungs [36], and was soon followed by similar demonstrations in human volunteers using polarized helium, both in the USA and in Germany [37, 38]. The striking difference between proton and ${ }^{3} \mathrm{He}$ chest images is illustrated in Fig. 5. Both images have been obtained in a 1.5 Tesla clinical
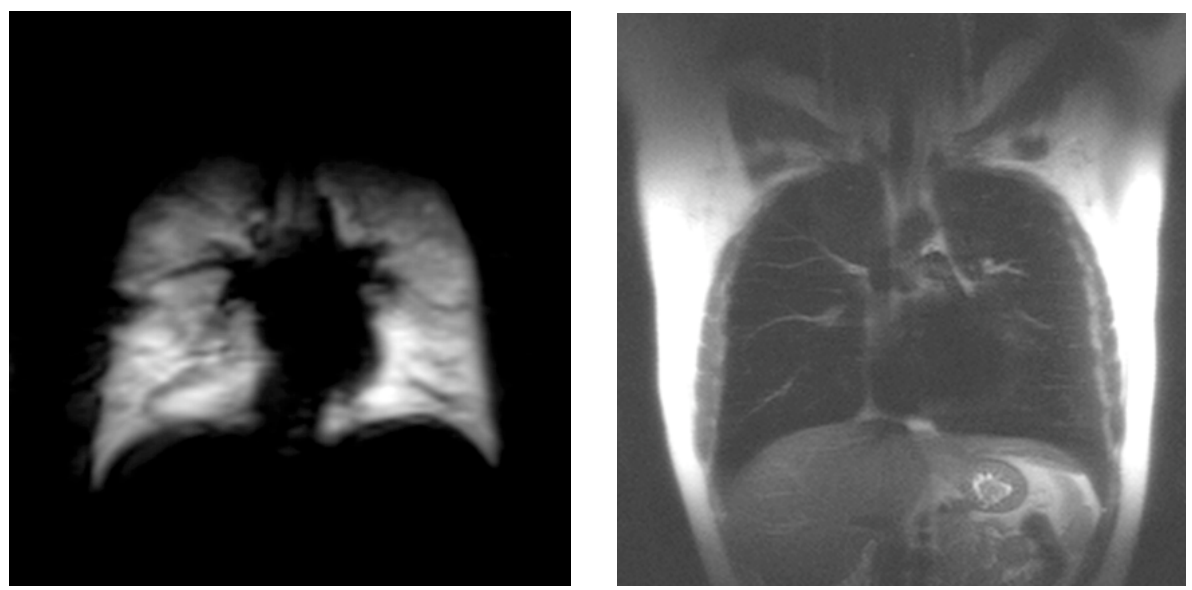

Figure 5. Chest MR images (1 cm thick slices) of the same normal subject acquired during breath hold. Left: FLASH ${ }^{3} \mathrm{He}$ image of the inhaled gas. Right: fast spin-echo proton image. The NMR frequency is changed from $49 \mathrm{MHz}$ for ${ }^{3} \mathrm{He}$ to $64 \mathrm{MHz}$ for protons. Courtesy of Jim Wild, Academic Radiology Dept., U. of Sheffield, $U K$.

MRI scanner, but using different coil systems operating at different frequencies (the gyromagnetic ratio of ${ }^{3} \mathrm{He}, \gamma_{\mathrm{He}} / 2 \pi=-32.44 \mathrm{MHz} / \mathrm{T}$, is $30 \%$ lower than that of $\mathrm{H})$. The left part of the figure displays a ${ }^{3} \mathrm{He}$ image recorded after the subject has inhaled a volume of order 0.3 liter of polarized gas in addition to a normal air intake. An intense signal is obtained from the lung airspaces in this slice, and a set of 15-20 such slices, recorded during a 10-12 s breath hold, allows to reconstruct a 3-D map of the gas distribution in the lungs. In this image, the space occupied by the heart is clearly visible, as well as a peripheral wedge-shape ventilation defect in the right lung, of a kind more often observed in asthmatic subjects. More details on the gas polarization process and on the specific imaging protocol will be given in the following. The proton image, on the right of Fig. 5, displays features that appear to be complementary. Indeed, intense signal now arises from parts of the chest that consist of tissues and contain no helium gas. But the striking feature is that the lung parenchyma appears to be dark, except 
for a few large blood vessels (corresponding to hypointense signal in the helium image). This is partly due to the low proton density of tissues, with airspaces occupying a significant fraction of the volume, but mostly results from the very short transverse relaxation time $T_{2}^{*}$ for protons in this highly heterogeneous tissue. Due to the magnetic susceptibility of the various components of the parenchyma, and to the small scales characterizing the alveolar structures $(0.1-0.3 \mathrm{~mm})$, strong internal magnetic field gradients resulting from the applied $1.5 \mathrm{~T}$ field very rapidly dephase the precessing magnetization, and this makes MRI of the lung a very difficult technical challenge [20]. Fortunately, these internal gradients less severely affect the precessing magnetization in the gas phase, thanks to the rapid diffusive movement of the gas atoms across alveoli that efficiently averages out spatial field variations at that scale. This motional averaging leads to NMR line narrowing, and $T_{2}^{*}$ for the gas is long enough $(>15 \mathrm{~ms}$ ) for imaging purposes.

As was briefly mentioned in section 2.2 , MRI of a low-density sample having the weak nuclear polarization (of order $10^{-5}$ ) that results from thermal equilibrium is not feasible for SNR reasons. For instance, the ${ }^{3} \mathrm{He}$ image in Fig. 5 has been obtained with a number density of helium of order $10^{18}$ atoms $/ \mathrm{cc}, 10^{5}$ times lower than that of protons in water: a high, out-of-equilibrium nuclear polarization, often referred to as hyperpolarization, is required to compensate for this decrease in density and thus provide a high enough magnetization for MRI purposes. In this section we first describe a few striking milestones in the development of hyperpolarization of noble gases, together with the motivations that drove these developments. Some specific features of MRI with polarized gases are then discussed, and selected topic in clinical research using lung MRI with polarized helium are finally outlined.

\subsection{Hyperpolarization of noble gases}

In the early days of optical pumping in atomic vapors [21, 22], no method had yet been found to polarize noble gases due to the lack of convenient optical transition from the atomic ground state. Still, as early as 1956, Walters and Fairbanks used a 1-D NMR imaging technique to study a phase-separation transition in liquid isotopic helium mixtures [39]. They filled a set of 3 connected reservoirs with liquid mixtures of ${ }^{3} \mathrm{He}$ and ${ }^{4} \mathrm{He}$ (see Fig. 6, left), and obtained 3 resolved NMR lines in the applied field gradient. The line intensities in the upper and lower reservoirs were interpreted as measurements of the ${ }^{3} \mathrm{He}$ concentration (with assumptions on magnetic susceptibility), and the coexisting concentration values were plotted as a function of the temperature (Fig. 6, right). Various other methods have been used since then to obtain this phase-separation diagram with improved accuracy, but this is probably the first MRI experiment with ${ }^{3} \mathrm{He}$, for which polarization was increased $(\times 100)$ by the use of low temperatures, and it was performed more than 50 years ago.

However, it is only with the development of optical pumping methods for noble gases that almost full nuclear polarization could be achieved. Optical pumping $(\mathrm{OP})$ is the redistribution of atoms among the energy sublevels of the ground 

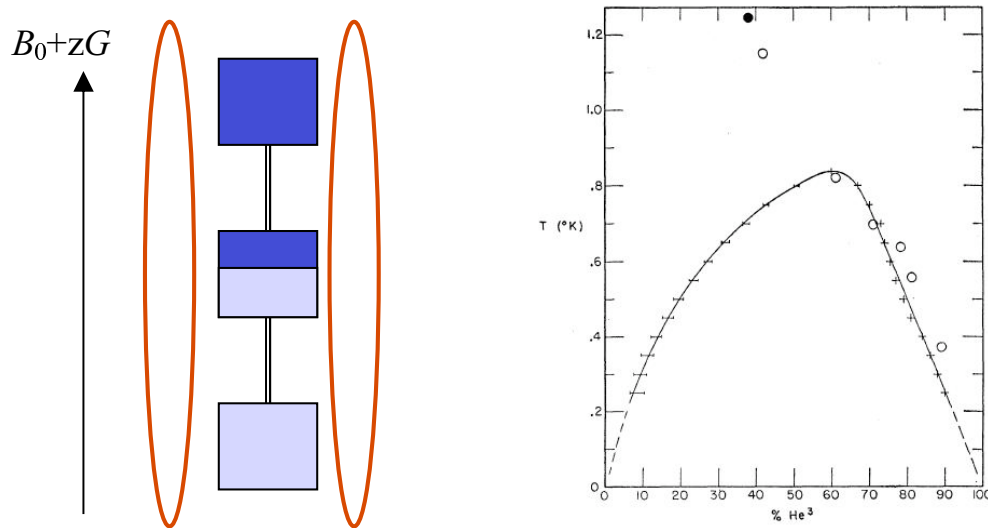

Figure 6. Early use of 1-D MRI in ${ }^{3}$ He to study phase-separation in liquid helium mixtures. Left: Principle of the experiment: the interface of the phase-separated mixture lies in the central part of the cell, while the upper and lower ends contain the ${ }^{3} \mathrm{He}$-rich and the dilute phases, respectively. The amplitudes of the frequencyresolved NMR lines from these parts of the cell are used to derive the ${ }^{3} \mathrm{He}$ concentrations Right: The coexisting concentrations, on the horizontal axis, are plotted for the explored temperature range (on the vertical axis), providing the first reported experimental phase-separation diagram in liquid helium mixtures (data from reference [39]).

state induced by resonant absorption of light [40]. In order to operate, OP thus requires an atomic system with (at least) two energy levels connected by an optical transition, and a light source tuned to this optical transition. The ground state (lower atomic level), and optionally the excited state (upper atomic level) have at least two sublevels, which may arise from fine or hyperfine structure terms, or from magnetic energy terms in the atomic Hamiltonian.

A very simple two-level system, used as a convenient illustrative model, is depicted in Fig. 7. Resonant light with circular polarization selectively depopulates one of the two sublevels of the ground state. Depending on the relative probabilities of spontaneous emission, a fraction of the re-emitted light takes away the angular momentum of the absorbed photon, which corresponds to inefficient OP cycles. The rest of the emitted photons have a linear $(\pi)$ polarization (vertical dotted arrows in Fig. 7), and a net quantum of angular momentum is deposited in the system. Indeed, the statistical properties of an ensemble of atoms are computed using a density operator formalism to describe the populations and quantum coherences of the sublevels; in most simple cases, only atomic populations are affected by OP processes, and simple rate equations can be derived. In this frame, 

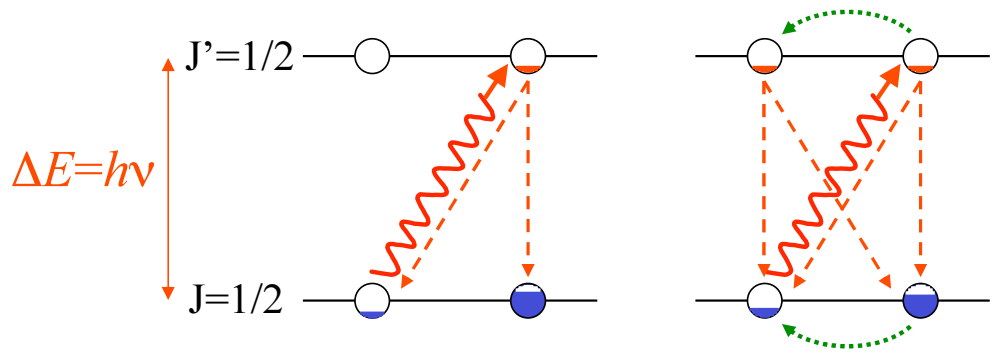

FiguRE 7. Example of OP processes in a two-level model system. The levels have angular momenta $J=J^{\prime}=1 / 2$, and each one contains two sublevels $m_{J}, m_{J^{\prime}}= \pm 1 / 2$. The sublevels are represented by circular symbols, more or less filled depending on their relative population. With a $\sigma_{+}$circular polarization of the OP light tuned to the optical transition frequency $\nu$, the transition $m_{J}$ $=-1 / 2 \rightarrow m_{J^{\prime}}=1 / 2$ (wavy arrow) is selectively excited. Spontaneous emission from the excited state (downwards dotted arrows) repopulates the sublevels of the ground state. Relaxation (population transfer between sublevels) is represented by additional dotted arrows in the right-hand side diagram.

the pumping rate scales with the incident light intensity as long as saturation effects can be neglected, i.e. typically below $10 \mathrm{~mW} / \mathrm{cm}^{2}$ to $1 \mathrm{~W} / \mathrm{cm}^{2}$ depending on the actual system.

When relaxation processes (additional arrows in the right hand side part of Fig. 7) efficiently transfer atoms between the sublevels of a given state, the overall OP efficiency can be significantly altered. Relaxation usually results from atomic collisions in the gas, and its rate strongly depends on the atomic state symmetry. For instance, even at moderate density (e.g. $10^{17} \mathrm{~cm}^{-3}$, or a few mbar at room temperature), relaxation rates can be higher than the radiative decay rates for a $\mathrm{P}$ or D state, but they are negligible for an S state. Assuming full redistribution of the atomic populations among the excited sublevels, the emitted light is unpolarized and the OP efficiency fully results from a depopulation mechanism, that can still be very efficient. In contrast, any relaxation in the ground state tends to reduce the OP efficiency; OP will thus easily and efficiently operate only if the atomic ground state is weakly affected by collisions, or at very low atomic densities (e.g. in an atomic beam).

As already mentioned, no convenient optical transition allows to perform OP on the ground state of any of the noble gases; among other reasons, they lie in the far-UV range. This is unfortunate since among their various isotopes, some of these atoms have a well-shielded, purely nuclear angular momentum. Especially for $I=1 / 2$, i.e. for ${ }^{3} \mathrm{He}$ and ${ }^{129} \mathrm{Xe}$, very long relaxation times (hours) of the nuclear polarization can be obtained in spite of frequent atomic collisions with other atoms 
or with adequately chosen cell walls. However, two indirect OP methods have been discovered in the 1960's to override this problem.

The first one, spin-exchange OP (SEOP) was demonstrated in 1960 [41], but at that time it only provided very low nuclear polarizations $(0.01 \%)$. Following this early demonstration, the reasons of its limited performance have been systematically studied, understood, and bypassed [42]. SEOP consists in performing OP on an alkali vapor mixed with a noble gas in a cell, usually Rb (see Fig. 8). Due to the
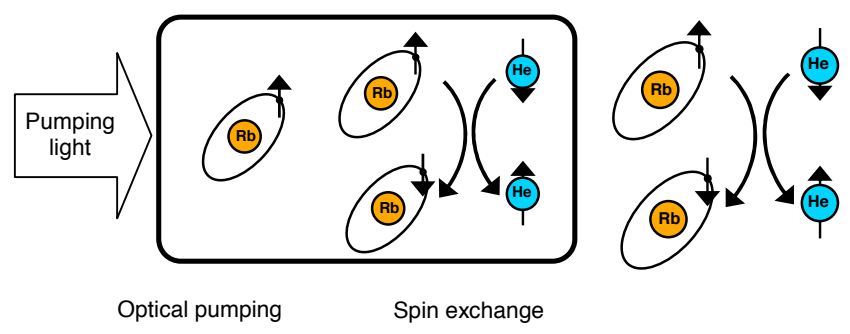

binary collision

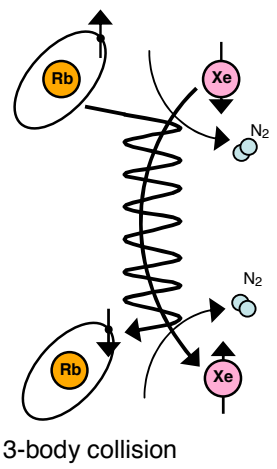

3-body collision

Figure 8. Spin-exchange OP in a $\mathrm{Rb}-{ }^{3} \mathrm{He}$ or ${ }^{129} \mathrm{Xe}$ mixture; $\mathrm{OP}$ is performed on the alkali atom, and its electronic orientation creates nuclear orientation of the noble gas during binary collisions (the dominant process for ${ }^{3} \mathrm{He}$ SEOP) or 3-body collisions (the dominant process for ${ }^{129} \mathrm{Xe}$ SEOP).

complicated structure of the atomic levels involved in the $\mathrm{OP}$ process (both $\mathrm{Rb}$ isotopes have a non-zero nuclear spin giving rise to hyperfine-structure sublevels), the OP process is not as simple as depicted in Fig. 7. Moreover, radiative decay from the excited state is advantageously quenched by collisions with added $\mathrm{N}_{2}$ gas to prevent reabsoption of unpolarized light. Still, using suitable powerful lasers for the $795 \mathrm{~nm}$ transition of $\mathrm{Rb}$, an efficient depopulation OP process allows to sustain a high degree of electronic polarization for the Rb atoms, that is transferred to the nuclei noble gas atoms during collisions via transient hyperfine coupling.

The second indirect method, metastability exchange OP (MEOP), was discovered in 1963 [43] and exclusively applies to ${ }^{3} \mathrm{He}$. It is interesting to note that in this first experiment, NMR measurements were performed and the optical detection of pulsed NMR was performed together with standard cw NMR (Fig. 9). This attention paid to NMR is probably due to the fact that this work was led by G.K. Walters, who conducted the NMR experiment depicted in Fig. 6 a few years before, and to the interest in magnetometry that motivated this research.

In a helium gas, MEOP is actually performed between two excited levels, the lower $2^{3} \mathrm{~S}$ level being metastable with a radiative lifetime of several thousands seconds (see Fig. 10). This level, which acts as a ground state for the OP process, is 

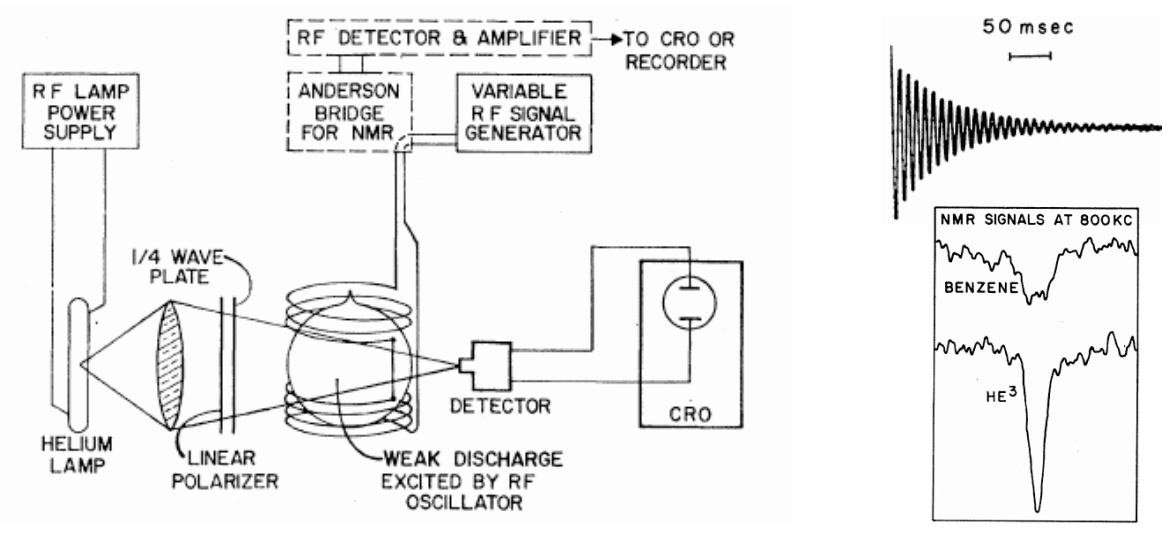

FiguRE 9. Sketch of the first MEOP experiment providing high nuclear polarization in ${ }^{3} \mathrm{He}$. Optical detection of NMR precession (top right) provided a much higher SNR than cw NMR (bottom right). Figures are from reference [43].

usually populated by electron collisions in a plasma discharge in a gas at moderate density $\left(10^{16}-10^{17} \mathrm{~cm}^{-3}\right)$. The lifetime of these metastable states is actually limited to $1-10 \mathrm{~ms}$ by diffusion to the cell walls, and their number density is of order $10^{-6}$ of the total density. For the ${ }^{3} \mathrm{He}$ isotope in this excited metastable state, an efficient coupling between the nucleus and the electrons (the hyperfine interaction) results in a strong entanglement of electronic and nuclear spins. Therefore the OPenforced optical orientation of the electronic angular momentum simultaneously induces nuclear orientation as well. This nuclear orientation is rapidly transferred to the atoms having remained in the ground state through metastability exchange collisions. This important collisional process takes place between the true ground state and the $2^{3} \mathrm{~S}$ metastable state, and corresponds to a very short interaction resulting in a fast exchange of the electronic excitations of the colliding atoms, with no change in the nuclear orientations.

Each of the two methods has its own advantages and limitations. For instance, SEOP operates both for ${ }^{3} \mathrm{He}$ and ${ }^{129} \mathrm{Xe}$, and is used for numerous studies involving polarized gases [44], e.g. in precise nuclear co-magnetometers in the search of the electric dipole moment of neutrons [45]. Progress in laser technology at $795 \mathrm{~nm}$, at first with Ti:Sapphire lasers, then with dedicated diode laser arrays, has made it an efficient tool for all applications requiring high-density polarized gases, such as spin filters for cold neutrons [46] or lung imaging [47]. To date, it is the only method to polarize xenon, of which large amounts can now be obtained with a high polarization (e.g. 0.3 liter/hour with $50 \%$ polarization [48]). For ${ }^{3} \mathrm{He}$, SEOP directly operates at high pressure (several bars), but is a slow process (several hours are required to obtain a sizable polarization). This difference originates from the much lower spin-exchange cross section that ensures polarization transfer from 


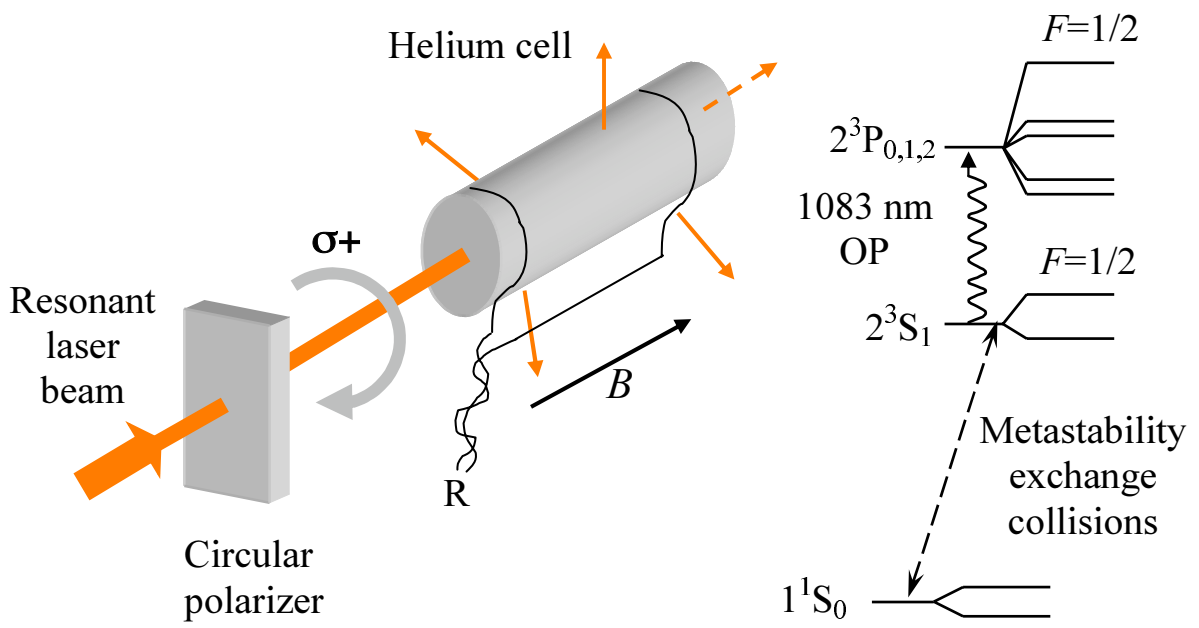

Figure 10. Left: schematic view of a MEOP setup. In a lowpressure helium-3 gas cell, a weak RF discharge promotes a small fraction $\left(\sim 10^{-6}\right)$ of the atoms into the excited metastable state $2^{3} \mathrm{~S}$, where resonant absorption of the circularly polarized $1083 \mathrm{~nm}$ light can occur. Nuclear polarization is transferred to the ground state atoms by metastability exchange collisions. Long cylindrical OP cells can be used thanks to the weak absorption of OP light, especially by a polarized gas. Right: atomic levels of ${ }^{3} \mathrm{He}$ and physical processes involved in the OP cycle. The $2^{3} \mathrm{~S}$ and $2^{3} \mathrm{P}$ states are composed of 2 and 5 sublevels, respectively, and the $1083 \mathrm{~nm}$ line has a complex structure. An efficient OP transition actually connects the highest-lying $F=1 / 2$ sublevels of the $2^{3} \mathrm{~S}$ and $2^{3} \mathrm{P}$ states, and the $\mathrm{OP}$ process is similar to that sketched in Fig. 7.

Rb to He., and current studies using mixtures of alkali atoms yield promising results [49].

When laser light with adequate spectral characteristics is used, MEOP provides very high nuclear polarization $(>70 \%)$ with good efficiency $(>1$ polarised nucleus per absorbed photon) [50]. With the recent development of spectrally suitable high power fiber lasers [51], the only drawback of this method is its limited range of operating pressures, of order 0.5-5 mbar, required to have a suitable plasma discharge in which metastable atoms are populated $[52,53]$. Whenever a higher final pressure is needed, non-relaxing compression of the gas is required. This is the case for several applications including lung MRI, and it introduces demanding requirements on the gas handling and compressing devices. However, it was recently shown that the range of operating pressures can be extended to 
several tens of mbar by performing MEOP in a high magnetic field, such as the $1.5 \mathrm{~T}$ field of an MRI system [54,55], which could make subsequent compression less difficult to perform.

The first attempt at compressing ${ }^{3} \mathrm{He}$ after it has been polarized by MEOP dates back to 1968 [56], using cryogenic means to increase the gas density and ultimately liquefy it. But only limited polarization (of order 1\%) could be obtained due to fast wall relaxation. It was only with the use of non-relaxing cryogenic coatings providing long $\mathrm{T}_{1} \mathrm{~s}$ [57] that polarized ${ }^{3} \mathrm{He}$ could be successfully cooled as a highly polarized gas, or liquid [58], allowing for instance studies of the effect of spin-polarization on quantum properties of gaseous ${ }^{3} \mathrm{He}[59,60]$. This series of studies in dense polarized systems obtained by cryogenic methods is currently focused on the non-linear NMR behavior that results from the action of distant dipolar fields in hyperpolarized liquids [61].

The following attempt at compressing polarized ${ }^{3} \mathrm{He}$ was made in 1970 using a mechanical Toepler pump (a mercury piston moving in a glass vessel) and only met with a limited success (less than $4 \%$ polarization at 0.3 bar) [62] due to the lack of powerful OP light sources. Mechanical compression attempts resumed only after the first multi-watt lasers for MEOP have been developed in the late 1980s [63], this time succeeding in reaching sufficient polarization and pressure for use in various experiments [64, 65], in particular to prepare polarized ${ }^{3} \mathrm{He}$ targets for the measurement of the neutron electric form factor in scattering experiments of polarized electron beams [66]. Since then, various non-relaxing mechanical compression techniques have been applied to polarized ${ }^{3} \mathrm{He}$, using a diaphragm pump [67], a peristaltic pump [68], or a piston compressor [69, 70] for applications in lung MRI, in more accurate electron scattering experiments, and in neutron spin filters [71, 72].

A convenient feature of MEOP-based polarization systems is the possibility to up- or down-scale the apparatus depending on requirements, and on available budget. Commercially available fiber lasers ${ }^{9}$ deliver up to $15 \mathrm{~W}$. The optimized large-scale system currently in operation at Mainz University, that is used to produce polarized gas for lung MRI, for scattering experiments and for neutron spin filters, makes use of five OP cells, each one being over $2 \mathrm{~m}$-long, to efficiently absorb as much as possible of the available pumping power [70]. With the high quantum efficiency of MEOP, this system routinely yields 1.2 bar $\times$ liter/hour of $80 \%$-polarized ${ }^{3} \mathrm{He}$, or 3.3 bar $\times$ litre/hour of $60 \%$-polarized ${ }^{3} \mathrm{He}[70]$. To date, systems with similar performance operate only at the ILL in Grenoble [69] and in Garching ${ }^{10}$ for neutron spin filters. For all applications, polarized ${ }^{3} \mathrm{He}$ gas must be shipped to the final user since these systems are too bulky and delicate to be

\footnotetext{
${ }^{9}$ We are aware of two companies having provided lasers at $1083 \mathrm{~nm}$ with the 2-GHz bandwidth matched to the Doppler absorption profile of He atoms: Keopsys (Lannion, France) and IPG Photonics (Burbach, Germany).

${ }^{10}$ The gas polarizer used in Garching was manufactured by IC-Automation, a Mainz-based company who built this sole system using the expertise of the research team of Mainz University. They have currently no plans to make more gas polarizing systems.
} 
transported. Storage cells suitable for transportation, i.e. with very long relaxation times and magnetic shielding to avoid loss of polarization, have been developed, and shipping of hundreds of liters of polarized gas has now been successfully performed [70]. More compact systems, in which cell size, laser power, and compressor flow rate are all consistently lower, have of course a significantly reduced performance, yielding e.g. 0.2-0.3 bar $\times$ liter/hour of $50 \%$-polarized ${ }^{3} \mathrm{He}[67,68]$. Still, for less demanding applications such as methodological developments in MRI, lung imaging in small animals, or imaging with a limited number of patients, these systems might offer an increased ease of use by providing locally-polarized gas on demand.

These options to gas provision must indeed be compared to the use of SEOPbased systems. In-house SEOP systems have been developed by several groups, and in addition a dozen commercial units ${ }^{11}$ have been implemented in selected hospitals for research on lung MRI. These systems routinely yield 1 liter of $30 \%$ polarized gas after 8-12 hours of OP, which is currently less than what both kinds of MEOP-based systems can provide.

\subsection{Specific features of imaging with polarized gases}

The most obvious difference between MRI with polarized noble gases and usual proton MRI is of course the lower operating frequency which results from the lower value of the gyromagnetic ratio $\left(30 \%\right.$ lower for ${ }^{3} \mathrm{He}, 3.6$ times lower for $\left.{ }^{129} \mathrm{Xe}\right)$. All rf systems, including transmit and receive coils, thus have to be designed for these unusual frequencies. However, more than ten years after the first lung image was obtained using prototype systems, commercial solutions now exist and operation at these frequencies is now a standard option for many systems.

The main difference that makes MRI with polarized gases so unusual for untrained MR physicists or radiologists is the non-renewable character of the polarization. Nuclear relaxation has to be considered as a plague, not as a natural way to obtain signals after just waiting a few seconds (the usual $T_{1}$ value for protons in tissues). Gas management has to carefully avoid relaxation losses at all stages, from the preparation of the polarized gas until the end of the image acquisition. Magnetic relaxation induced by walls of gas containers is now well understood and controlled [73, 74, 75], and all materials in contact with the gas (tubes, valves, mouthpieces or respiratory masks...) must be selected with care. Magnetic relaxation can also occur in the bulk of the gas due to atomic diffusion in inhomogeneous magnetic field, with a typical rate:

$$
1 / T_{1}^{\text {magn. }} \sim D(G / B)^{2}
$$

\footnotetext{
${ }^{11}$ The polarizers have been developed on the late 1990s by MITI, Magnetic Imaging Technologies, Inc., a spinoff company from the Princeton Group that was involved in the first lung imaging demonstration [36]. MITI was then sold to Nycomed-Amersham, which later became Amersham Health, which in turn was later sold to GE Healthcare. The polarizers are still maintained in operation, but their future status is unknown.
} 
that scales with the diffusion coefficient $D\left(2 \mathrm{~cm}^{2} / \mathrm{s}\right.$ at 1 bar for $\left.{ }^{3} \mathrm{He}\right)$ and with the square of the relative field inhomogeneity $(G / B$ in the case of a uniform gradient $G)$. This shows in particular that polarized gas should not dwell in parts of the MRI magnet where the field has a steep variation with position. A last important source of relaxation lies in collisions with the paramagnetic $\mathrm{O}_{2}$ molecules of air, which induce a decay rate:

$$
1 / T_{1}^{\text {magn. }} \sim 0.41 \times p \mathrm{O}_{2}
$$

where $p \mathrm{O}_{2}$ stands for the partial pressure of $\mathrm{O}_{2}$ (in bars). This value is inferred from reference [76] for room temperature, and corresponds to a relaxation time of $11.4 \mathrm{~s}$ in room air (with $21 \% \mathrm{O}_{2}$ ). Polarized ${ }^{3} \mathrm{He}$ must thus be preserved from exposure to air until the time at which it is inhaled. Polarization decays rather rapidly in-vivo, and data acquisition must be performed in a matter of seconds - which anyhow usually corresponds the the maximum time patients with respiratory problems can hold their breaths. This relaxation process is indeed a constraint, but can also be used to evaluate the $\mathrm{O}_{2}$ contents of the lungs (see Fig. 14 in the next section). The last feature of imaging with a hyperpolarized sample is that the sequence of rf pulses must be tailored for an optimal use of the available magnetization, either by an appropriate choice of successive small tip angles, or by using multiple echo acquisition whenever possible. Since polarized gas is not easily available, and so far only in rather limited amounts, a careful planning of the patients' imaging sessions is necessary.

A second specific series of features of MRI with polarized gases originates from the fast diffusion of atoms in a gas phase. The beneficial consequence on transverse relaxation induced by internal gradients has already been discussed at the beginning of section 4, but adverse effect of diffusion also exist. For instance, the spatial resolution is intrinsically limited by diffusion: for a given amplitude $G$ of an imaging gradient, the spatial resolution is $\delta x=1 / \gamma G T_{\text {obs }}$ (see section 3.1), while diffusion-induced attenuation limits the useful time to $T_{\text {obs }} \sim D \delta x^{2}$. The maximum resolution thus scales as $\delta x \sim(D / \gamma G)^{1 / 3}$. For standard MRI systems, for which $G$ cannot exceed $30 \mathrm{mT} / \mathrm{cm}$, the highest resolution for ${ }^{3} \mathrm{He}$ diffusing in air is of order $0.4 \mathrm{~mm}$, not quite small enough to directly image alveolar structures: MR microscopy is not possible with gases. However diffusion weighting in MRI offers the potential to probe gas diffusion over a wide range of time and paces scales, linked by the same relation that directly limits image resolution: $T_{\text {obs }} \sim D \delta x^{2}$. Characterizing apparent diffusion at various scales within the lung may indirectly provides relevant information on the lung microstructure.

A last specific feature of NMR or MRI with polarized samples is that the SNR is field-independent over a wide range of field values $B_{0}$ (see section 2.2). The direct influence of $B_{0}$ is limited to the effect of internal gradients on the signal characteristics. From this point of view, high fields (1.5 T and above) induce short values for $T_{2}^{*}$, that may be more sensitive to the alveolar characteristic size and shape, thus providing a physiologically relevant contrast mechanism [77]. Conversely, transverse relaxation times are longer at reduced fields, as demonstrated 
for instance at $0.1 \mathrm{~T} \mathrm{[78]} \mathrm{and} \mathrm{at} 3 \mathrm{mT}$ [79]. Weaker field gradients can then be used for imaging or for ADC measurements. This actually improves SNR in images and allows measuring diffusion coefficients at longer time scales, thus providing more information on the connectivity of lung air spaces. Monitoring the decay of spinecho trains provides a precise way of measuring ADC coefficients in this case. At ultra-low fields, internal gradients are so low that the observed decay in spin-echo experiments results from the oxygen-induced relaxation process for which Eq. 13 also applies [79].

Another clear advantage of operating at low field is the reduced cost and increased flexibility of the imaging system: an open geometry can be designed, and standing or sitting patients may be examined. Initial studies at ultra low field in home made vertical scanners have demonstrated the potential of the technique $[80,81]$. This could open up possibilities of low-cost dedicated scanners for the screening or follow-up of lung diseases.

\subsection{Current status of research}

In this section we present a selection of results chosen to illustrate the potential of lung MRI in humans with polarized ${ }^{3} \mathrm{He}$, deliberately overlooking all the important work done using ${ }^{129} \mathrm{Xe}$ or in animal models, for which much information can be found in the literature (e.g. [82]). These results have been obtained by clinical research teams participating in the PHIL project (Polarized Helium to Image the Lung), a joint effort of nine European research teams in five countries [83]. An important objective of the project was to demonstrate the potential and the validity of the new MRI method as a diagnostic and prognostic tool for given lung pathologies: emphysema and selected Chronical Obstructive Pulmonary Diseases (COPD), such as bronchitis and bronchiolitis. This choice was motivated by the frequent occurrence of these diseases and the very high cost of their treatment for society: $10 \%$ of the population and $25 \%$ of the smokers suffer from COPD, which is the fourth cause of mortality in Europe and in the USA. The core of the project was to perform a clinical trial on a large group of patients with the ${ }^{3} \mathrm{He}$ MRI method and with conventional techniques: pulmonary function tests, High Resolution Computed Tomography (HRCT), Krypton scintigraphy. An important objective of the PHIL project was to provide new tools for the study of COPD, aiming at differentiation of various types of diseases, as well as their detection at an early stage, with expectation that in the long range the findings of the project could lead to monitoring therapeutic treatment. The clinical trial successfully enrolled a total of 116 subjects (62 COPD, 17 alpha-1-antitrypsin deficiency (ATD) and 37 healthy volunteers). The collaborative work has triggered the dissemination of the method in Europe. In addition to the trials performed in Mainz, Sheffield and Copenhagen, animal model studies have been made in Lyon and Madrid, and methodological developments in Paris area, Cracow and Mainz. The collaboration is currently pursued, including several new participants, in the Phelinet project that aims at promoting the training of young researchers and scientific exchanges between participants [84]. 
An important objective of research with ${ }^{3} \mathrm{He}$ MRI is the systematic comparison of MR images with other existing imaging modalities. Sets of images obtained by ${ }^{3} \mathrm{He}$ ventilation images obtained during $12 \mathrm{~s}$-long breath holds and by ${ }^{81 \mathrm{~m}} \mathrm{Kr}$ single photon emission computed tomography (SPECT) ${ }^{12}$ are displayed in Figs. 11 (for a normal volunteer) and 12 (for a COPD patient). In both sets of images,
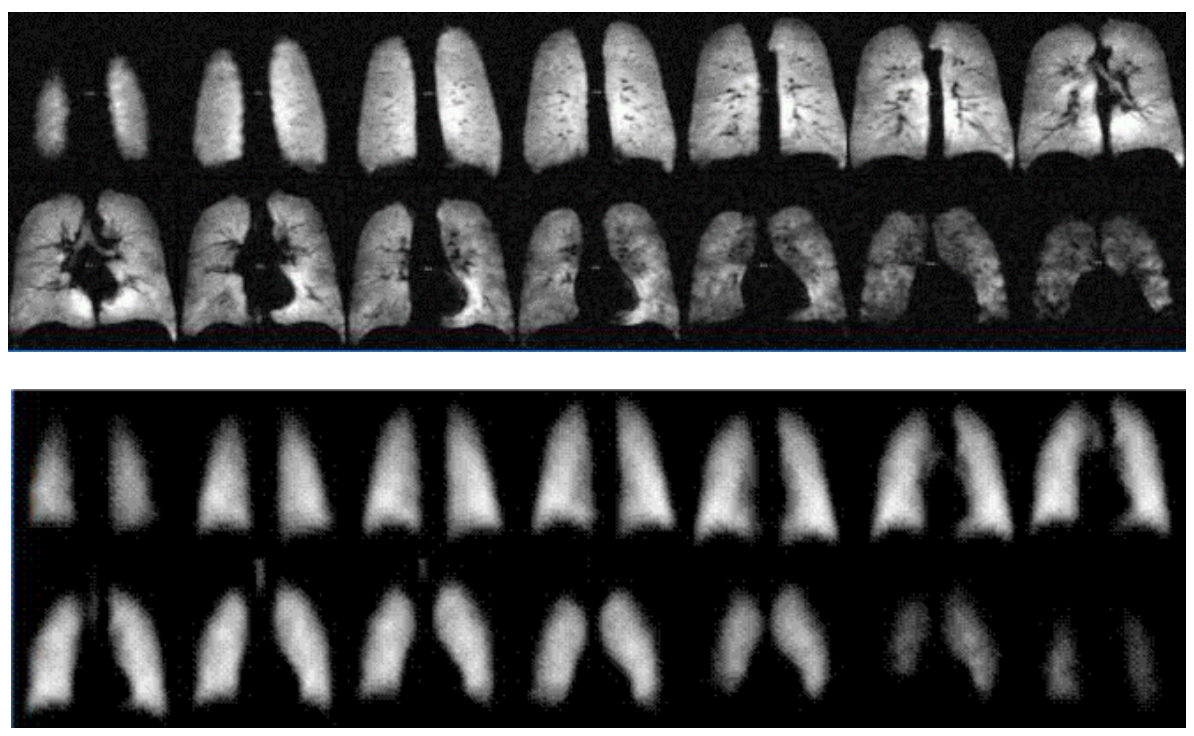

FiguRE 11. Lung images in a normal volunteer: ${ }^{3} \mathrm{He}$ MRI (top), and ${ }^{81 \mathrm{~m}} \mathrm{Kr}$ SPECT (bottom).- Coronal slices from the posterior part of the lung to the anterior part, from the upper left corner. Courtesy of Trine Stavngaard and Lise Vejby Soegaard, Danish Research Centre for Magnetic Resonance, Copenhagen University Hospital, Hvidovre, Denmark.

the spatial resolution provided by ${ }^{3} \mathrm{He} \mathrm{MRI}$ is far superior $\left(1.3 \times 1.3 \mathrm{~mm}^{2}\right.$ in each $10 \mathrm{~mm}$-thick slice), and much more detailed information is provided on ventilation defects in Fig 12. The study included 32 patients, and showed good correlation between the ventilation defects assessed by the two methods, in spite of the difference in lung inflation conditions for the two methods (17 min.-long SPECT images were acquired close to tidal volume during normal breathing) [85].

\footnotetext{
${ }^{12} \mathrm{~A}$ cyclotron produces the radioactive isotope ${ }^{81} \mathrm{Rb}$, that decays to metastable ${ }^{81 \mathrm{~m}} \mathrm{Kr}$, which the patient inhales. The metastatable isotope decays and emits gamma photons that are detected. A gamma camera records planar projections (scintigrams) showing the ventilated area in the lung. From several scintigrams taken from various angles, a 3-D reconstruction of the lung ventilation is obtained. For the images in Figs 11 and 12, the spatial resolution was $8 \mathrm{~mm}$, the radiation dose was $1 / 2 \mathrm{mSv}$, and the imaging time was $17 \mathrm{~min}$.
} 

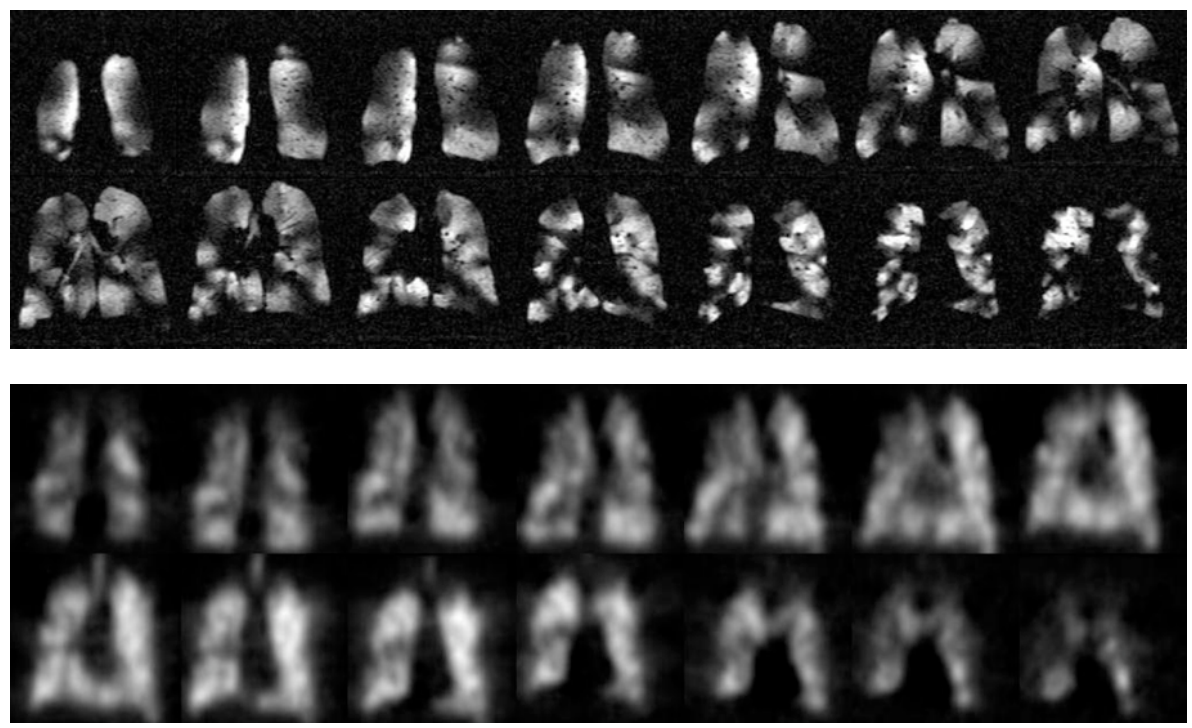

Figure 12. Same ${ }^{3} \mathrm{He}$ MRI and ${ }^{81 \mathrm{~m}} \mathrm{Kr}$ SPECT lung images as in Fig. 11 for a COPD patient. Courtesy of Trine Stavngaard and Lise Vejby Soegaard.

Much higher time-resolution can be obtained using a sliding window radial acquisition scheme [86]. Projection images obtained by this method are shown in Fig. 13, with a time interval between displayed images of $0.54 \mathrm{~s}$. For the normal volunteer, the temporal passage of gas down the trachea, into the bronchi and peripheral lung is clearly resolved. For the COPD patient, the image series shows regions of ventilation obstruction in both lungs, particularly in the upper lobes, and a delayed emptying/depolarization of gas in the lower left lobe which could be indicative of air trapping. Such dynamic ventilation maps may provide valuable information on ventilation defects in COPD patients, complementary to the static ventilation defect data obtained during a breath hold (Figs. 11 and 12) [87].

Decay of signal during a breath hold resulting from $\mathrm{O}_{2}$-induced relaxation (Eq. 13) can be recorded in a series of images, from which maps of the local partial pressure of oxygen are computed, as displayed in Fig. 14 for a normal subject and a COPD patient [88]. When low-resolution images are acquired, $p \mathrm{O}_{2}$ values are determined with such high accuracy that their time-evolution provides information on regional oxygen uptake. It is dependent on lung perfusion, and thus the uptake map can be seen as a regional ventilation perfusion $\left(\mathrm{V}_{\mathrm{A}} / \mathrm{Q}\right)$ map of the lung [89], allowing a direct measurement of the regional $\mathrm{V}_{\mathrm{A}} / \mathrm{Q}$ distribution in a noninvasive fashion at relatively high resolution when compared to ventilation perfusion scintigraphy. 


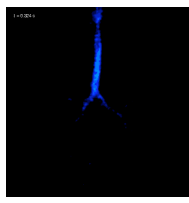

$\mathrm{t}=0.32 \mathrm{~s}$

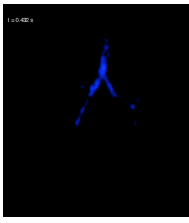

$\mathrm{t}=0.43 \mathrm{~s}$

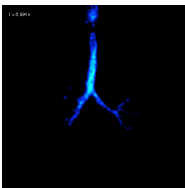

$\mathrm{t}=0.59 \mathrm{~s}$

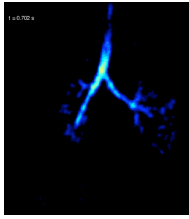

$\mathrm{t}=0.70 \mathrm{~s}$

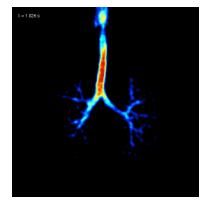

$\mathrm{t}=1.03 \mathrm{~s}$

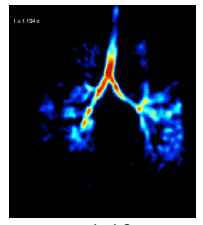

$\mathrm{t}=1.13 \mathrm{~s}$

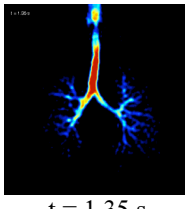

$\mathrm{t}=1.35 \mathrm{~s}$

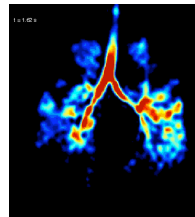

$\mathrm{t}=1.62 \mathrm{~s}$

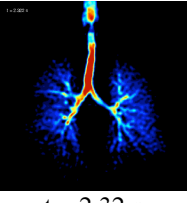

$\mathrm{t}=2.32 \mathrm{~s}$

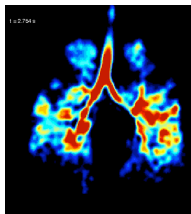

$\mathrm{t}=2.75 \mathrm{~s}$

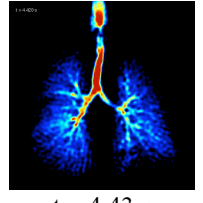

$\mathrm{t}=4.43 \mathrm{~s}$

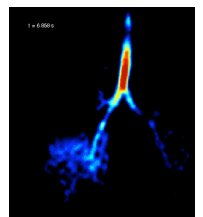

$\mathrm{t}=6.86 \mathrm{~s}$

FIGURE 13. Dynamic projection images obtained using a radial sequence (time resolution: $0.135 \mathrm{~s}$ ). Top: selected images in a series from a healthy normal subject recorded during an inhalation of $300 \mathrm{ml}$ of $40 \%$-polarized ${ }^{3} \mathrm{He}$ gas. Bottom: Similar dynamic time series from a COPD patient. Courtesy of Jim Wild, Academic Radiology Dept., U. of Sheffield, UK.

Figure 15 displays exampled of ADC maps obtained using a bipolar diffusionsensitization gradient, a standard technique which provides information on diffusion of ${ }^{3} \mathrm{He}$ atoms over relatively short times and distances. Such images and the related histograms of increased ADC values have been shown to correlate well with enlarged alveolar sizes of patients with emphysema [90].

These few sets of images have been chosen as illustrative examples of the various relevant physiological information that MRI with polarized ${ }^{3} \mathrm{He}$ may quantitatively provide. Similar research has been indeed performed since the early days of polarized gas MRI also in the USA, where the University of Virginia has been, and remains, one of the most active research centers. It is pursued by tens of teams worldwide, including in Canada and in Japan, who are involved in lung MRI with polarized ${ }^{3} \mathrm{He}$ or ${ }^{129} \mathrm{Xe}$. More than 1000 patients have now been subject to various imaging protocols, and the potential of MRI in the early detection and in the staging of disorders such as asthma, emphysema, and cystic fibrosis has been explored. Its potential interest has also been suggested for very different problems such as the follow-up of lung transplants, or the comparison of lung development during childhood for normal children [91] and for children with a pre-term birth history [92].

\section{Conclusion and prospects}

Looking back at the history of NMR and of MRI, one is impressed by its fast development as a research field, as a tool, as an industrial opportunity, and as 

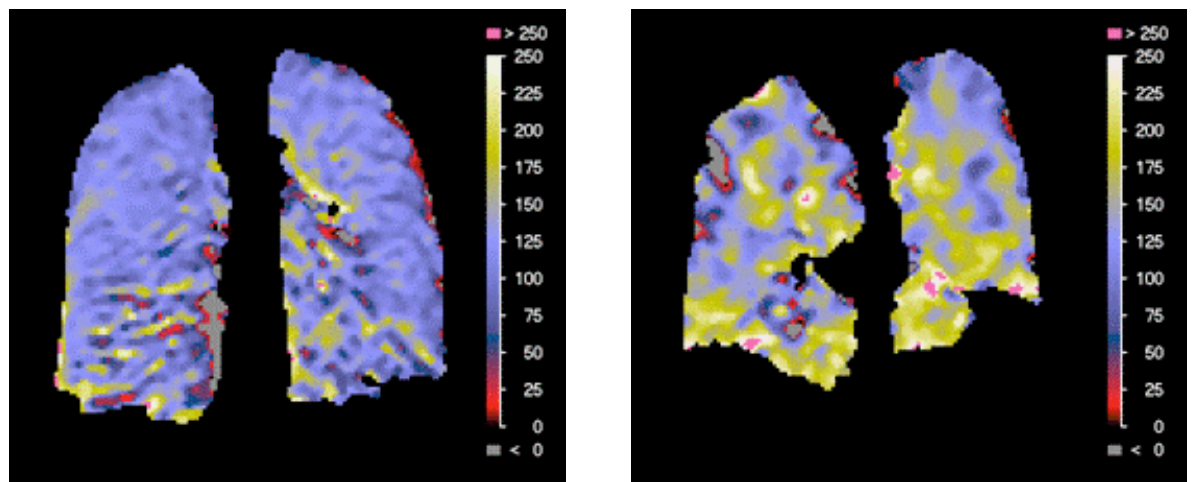

FiguRE 14. Measurement of oxygen concentration in the lung using ${ }^{3}$ He imaging. Left: Image of a healthy volunteer with relatively homogeneous distribution of oxygen. Right: Image of a patient with chronic obstructive pulmonary disease (COPD) demonstrating inhomogeneous distribution of oxygen because of regional impairment of oxygen uptake by blood. Thus, alveolar oxygen concentration remains elevated in these regions when compared with regions with normal ventilation and perfusion. Data from reference [88] - Courtesy of Wolfgang Schreiber, Johannes GutenbergUniversity Mainz, Medical School, Germany.

widespread clinical imaging modality. NMR moved rapidly from fundamental research at Stanford University to commercialization because of Varian's involvement. From that point, industry led the development, that depended more on technology than on fundamental research. Later on, through most of MRI's evolution, the challenges were to improve image quality and to reduce scan times, which again involved a lot of technology. As regards MRI, every decade was marked by spectacular qualitative and quantitative progress. Following the physicists' early demonstrations in the $70 \mathrm{~s}$, leading instrumentation companies heavily invested in research and development in the $80 \mathrm{~s}$, and started clinical evaluation of images. The $90 \mathrm{~s}$ were the time of the increase in available equipment for routine use, and of the growing clinical usefulness of the images. In the recent years, together with a steady increase in the number of operational imaging sites, there is an impressive reduction of scan times with the use of revolutionary methods for data acquisition and signal processing.

It can be argued that one key factor for this success story probably lies in its excellent timing [93]. For instance, two decades earlier, the technology for wholebody superconducting magnets and, more importantly, for computers performing all the necessary Fourier transforms, simply did not exist. Two decades later, 

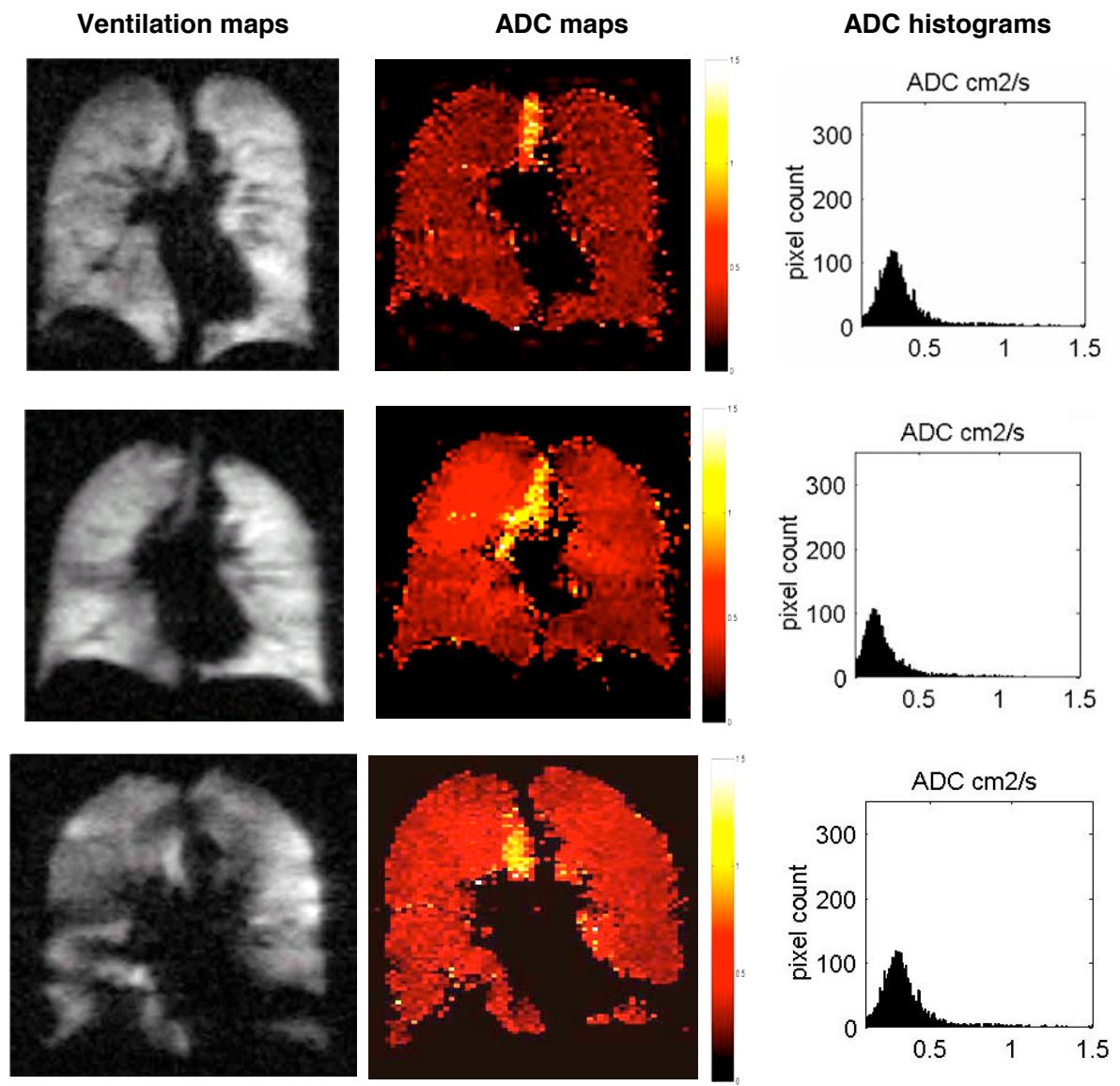

FIGURE 15. Left to right: ${ }^{3} \mathrm{He}$ MRI gas density projection images, ADC maps and ADC histograms. Top to bottom: data from a 48year-old female healthy non-smoker, a 51-year-old female healthy smoker, and a 62-year-old male diagnosed COPD patient. Courtesy of J. Wild, Academic Radiology Dept., U. of Sheffield, UK.

the demand for diagnostic imaging would have been less compelling, and betterestablished imaging modalities using well-controlled ionizing radiations (e.g. CTscans) would possibly have been considered as sufficient golden standards. Both the equipment industry and the radiologic profession would probably have required a strong incentive to accept such innovative methods and techniques. But even more important is the fact that the regulatory environment has drastically changed since the $80 \mathrm{~s}$. With the increasing concern regarding the impact of static and oscillatory fields, the level of proof of safety required for new instruments based 
on new principles is now tremendous. As a result, the development costs until the obtention of FDA approval in the USA, and of the equivalent certification in other countries, has become so high that it would probably be impossible to fund, neither by industry, who now have limited freedom for such ventures, nor by academic institutions, who have increasingly limited budget for unscheduled research.

The history of the new MRI technique for imaging the lung - the only organ that conventional MRI can hardly observe - has in a way followed a parallel, or rather a delayed path. Like NMR, it appeared as an unexpected outcome of decades of research and of instrumental developments aiming at totally different fundamental studies with highly polarized helium-3. With optical pumping, the stage was set since the $60 \mathrm{~s}$, but the main character in the story - the powerful laser needed for sufficient gas polarization - delayed its appearance until the 90s. Up to now, access to polarized gas has been difficult and this remains the main bottleneck preventing many research groups to become active in the field. The technology exists, it has been demonstrated for both optical pumping methods, including with a small number of commercial systems, and yet it is not widely developed. The arguments given by potential manufacturers include the uncertain status of patents regarding MRI with polarized gases, the increasing difficulty and cost of certification processes: since gases are inhaled, they are considered as drugs, and approval is consequently much harder to obtain than for a new type of coil or of MR sequence. Such arguments are disturbingly reminiscent of the above uchronic discussion on proton MRI.

In spite of these differences in timing, both proton MRI and polarized gas MRI are incredibly versatile imaging modalities with a unique potential for diagnosis. They are totally non-invasive, they can be rugged tools for the clinician or powerful instruments for the most advanced research - one may think, for instance, of the studies of cognitive processes using functional MRI of the brain. The remaining challenges for the dissemination and use of helium-3 MRI are now probably off the hands of the physicists who contributed to the beginning of this story, but they all wish it will have a happy end.

\section{References}

[1] I.I. Rabi, S. Millman, P. Kusch, and J. R. Zacharias, A new method of measuring nuclear magnetic moments, Phys. Rev. 53 (1938), 318; I.I. Rabi, S. Millman, P. Kusch, and J. R. Zacharias, The molecular beam resonance method for measuring nuclear magnetic moments. The magnetic moments of Li6, Li7 and F19, Phys.Rev. 55 (1939), 526.

[2] E.M. Purcell, H.C. Torrey, and R.V. Pound, Resonance absorption by nuclear magnetic moments in a solid, Phys. Rev. 69 (1946), 37.

[3] F. Bloch, W. W. Hansen, and M. Packard, Nuclear induction Phys. Rev. 69 (1946), 127; F. Bloch, W. W. Hansen, and M. Packard, The nuclear induction experiment, Phys. Rev. 70 (1946), 474. 
[4] F. Bloch, Nuclear induction, Phys. Rev. 70 (1946), 460.

[5] H.C. Torrey, Transient nutations in nuclear magnetic resonance, Phys. Rev. 76 (1949), 1059.

[6] E.L. Hahn, Nuclear induction due to free Larmor precession, Phys. Rev. 77 (1950), 297.

[7] E.L. Hahn, Spin echoes, Phys. Rev. 80 (1950), 580.

[8] J.T. Arnold, S.S. Dharmatti, and M.E. Packard, Chemical effects on nuclear induction signals from organic compounds, J. Chem. Phys. 19 (1951), 507.

[9] C.J. Gorter and L.J.F. Broer, Negative result of an attempt to observe nuclear magnetic resonance in solids, Physica 9 (1942), 591.

[10] R.J. Singer, Blood-flow rates by NMR measurements, Science 130 (1959), 1652.

[11] J.A. Jackson and W.H. Langham, Whole-body NMR spectrometer, Rev. Sci. Instrum. 39 (1968), 510.

[12] P.C. Lauterbur, Image formation by induced local interactions: examples of employing nuclear magnetic resonance, Nature 242 (1973), 190.

[13] A.N. Garroway, P.K. Grannell, and P. Mansfield, Image formation in NMR by a selective irradiative process, J. Phys. C 7 (1974), L457.

[14] P. Mansfield and A.A. Maudsley, Planar spin imaging by NMR, J. Magn. Reson. 27 (1977), 101.

[15] R.R. Ernst and W.A. Anderson, Application of Fourier transform spectroscopy to magnetic resonance, Rev. Sci. Instrum. 37 (1966), 93.

[16] A. Kumar, D. Welti, and R.R. Ernst, NMR-Fourier-Zeugmatography, J. Magn. Reson. 18 (1975), 69.

[17] W.A. Edelstein, J.M.S. Hutchison, G. Johnson and T.W. Redpath, Spin warp NMR imaging and applications to human whole-body imaging, Phys. Med. Biol. 25 (1980), 751.

[18] From the MEDLINE database of the U.S. National Library of Medicine. 4600 international journals in all areas of the life sciences, with particular emphasis on biomedicine are indexed.

[19] S. Gleyzes, et al., Quantum jumps of light recording the birth and death of a photon in a cavity, Nature 446 (2007), 297.

[20] E.D. Pracht, J.F.T Arnold, T.T. Wang, et al., Oxygen-enhanced proton imaging of the human lung using T2*, Magn. Reson. Med. 53 (2005), 1193.

[21] A. Kastler, Optical methods of atomic orientation and of magnetic resonance, J. Opt. Soc. Am. 47 (1957), 460.

[22] B. Cagnac, J. Brossel and A. Kastler, RMN du mercure Hg-201 aligné par pompage optique, C.R Acad. Sci. 246 (1958), 1827.

[23] J. Jeener, Equivalence between the "classical" and the "Warren" approaches for the effects of long range dipolar couplings in liquid NMR, J. Chem. Phys. 112 (2000), 5091.

[24] D.I. Hoult and P.C. Lauterbur, The sensitivity of the zeugmatographic experiment involving human samples, J. Magn. Reson. 34 (1979), 425. 
[25] J. Bittoun, B. Querleux and L. Darrasse, Advances in MR imaging of the skin, NMR Biomed. 19 (2006), 723; L. Darrasse and J.-C. Ginefri, Perspectives with cryogenic RF probes in biomedical MRI, Biochimie 85 (2003), 915.

[26] M. Mossle, et al., SQUID-detected in vivo MRI at microtesla magnetic fields, IEEE Transactions on Applied Superconductivity 15 (2005), 757.

[27] D. Budker and M. Romalis, Optical magnetometry, Nature Physics 3 (2007), 227.

[28] H.J. Mamin, M. Poggio, C.L. Degen and D. Rugar, Nuclear magnetic resonance imaging with 90-nm resolution, Nature Nanotechnology 2 (2007), 301.

[29] J. Pauly, P. Le Roux, D. Nishimura and A. Macovski, Parameter relations for the Shinnar-Le Roux selective excitation pulse design algorithm, IEEE Trans. Med. Imaging 10 (1991), 53.

[30] G. McGibney, M.R. Smith, S.T. Nichols and A. Crawley, Quantitative evaluation of several partial Fourier reconstruction algorithms used in MRI, Magn. Reson. Med. 30 (1993), 5159.

[31] D.K. Sodickson and W.J. Manning, Simultaneous acquisition of spatial harmonics (SMASH): Fast imaging with radiofrequency coil arrays, Magn. Reson. Med. 38 (1997), 1603.

[32] K.P. Pruessmann, M. Weiger, M.B. Scheidegger and P. Boesiger, SENSE: Sensitivity encoding for fast MRI, Magn. Reson. Med. 42 (1999), 2962.

[33] M. Lustig, D. Donoho and J.M. Pauly, Sparse MRI: The application of compressed sensing for rapid MR imaging, Magn. Reson. Med., in press, DOI: 10.1002/mrm.21391 (2007).

[34] M. Goldman, H. Johannesson, O. Axelsson and M. Karlsson, Hyperpolarization of 13C through order transfer from parahydrogen: a new contrast agent for MRI, Magn. Reson. Imaging. 23 (2005), 153.

[35] S. Mansson, et al., 13C imaging-a new diagnostic platform, Eur Radiol. 16 (2006), 57.

[36] M.S. Albert, G.D. Cates, B. Driehuys, et al., Biological MRI using laser-polarized 129Xe, Nature 370 (1994), 199.

[37] J.R. MacFall, H.C. Charles, R.D. Black, et al., Human lung air spaces: potential for MRI with hyperpolarized 3He, Radiology 200 (1996), 553.

[38] H.U. Kauczor, D. Hofmann, K.F. Kreitner, et al., Normal and abnormal pulmonary ventilation: Visualization at hyperpolarized 3He MRI, Radiology 201 (1996), 564.

[39] G.K. Walters and W.M. Fairbank, Phase separation in 3He-4He solutions, Phys. Rev. 103 (1956), 262.

[40] A. Kastler, Méthodes optiques d'étude de la résonance magnétique, Physica, 17 (1951), 191.

[41] M.A. Bouchiat, T.R. Carver and C.M. Varnum, Nuclear Polarization in He3 Gas Induced by Optical Pumping and Dipolar Exchange, Phys. Rev. Lett. 5 (1960), 373.

[42] W. Happer, Optical Pumping, Rev. Mod. Phys. 44 (1972), 169.

[43] F.D. Colegrove, L.D. Schearer and G.K. Walters, Polarization of He3 gas by optical pumping, Phys. Rev. 132 (1963), 2561.

[44] T.G. Walker and W. Happer, Spin-exchange optical pumping of noble-gas nuclei, Rev. Mod. Phys. 69 (1997), 629. 
[45] D. Bear, et al., Limit on Lorentz and CPT violation of the neutron using a twospecies noble-gas maser, Phys. Rev. Lett. 85 (2000), 5038 - erratum in Phys. Rev. Lett. 89, 209902.

[46] G.L. Jones, et al., Test of He-3-based neutron polarizers at NIST, Nuc. Instr. Meth. A 440 (2000), 772.

[47] T. Chupp and S. Swanson, Adv. At. Mol. Opt. Phys. 45 (2001), 51.

[48] I.C. Ruset, S. Ketel and F.W. Hersman, Optical pumping system design for large production of hyperpolarized 129Xe, Phys. Rev. Lett. 96 (2006), 053002.

[49] W.C. Chen, T.R. Gentile, T.G. Walker, et al., Spin-exchange optical pumping of He-3 with Rb-K mixtures and pure K, Phys. Rev. A 75 (2007), 013416.

[50] P.J. Nacher and M. Leduc, Optical pumping in 3He with a laser, J. Phys. (Paris) 46 (1985), 2057.

[51] G. Tastevin, S. Grot, E. Courtade, S. Bordais and P.-J. Nacher, A broadband ytterbium-doped tunable fiber laser for 3He optical pumping at 1083 nm, Applied Physics B 78 (2004), 145.

[52] M. Leduc, S.B. Crampton, P.J. Nacher and F. Laloe, Nuclear polarization of gaseous 3He by optical pumping, Nuclear Sci. App. 1 (1983), 1.

[53] P.-J. Nacher, E. Courtade, M. Abboud, A. Sinatra, G. Tastevin and T. Dohnalik, Optical pumping of helium-3 at high pressure and magnetic field, Acta Phys. Polon. B 33 (2002), 2225. Online at http://hal.archives-ouvertes.fr/hal-00002223/

[54] M. Abboud, A. Sinatra, X. Maitre, G. Tastevin and P.-J. Nacher, High nuclear polarization of $3 \mathrm{He}$ at low and high pressure by metastability exchange optical pumping at 1.5 Tesla, Europhys. Lett. 68 (2004), 480.

[55] A. Nikiel, T. Palasz, M. Suchanek, et al., Metastability exchange optical pumping of 3 He at high pressure and high magnetic field for medical applications, Eur. Phys. J. Special Topics 144 (2007), 255.

[56] H.H. Mc Adams, Dynamic nuclear polarization of liquid 3He by optical pumping, Phys. Rev. 170 (1968), 276.

[57] R. Barbe, F. Laloe and J. Brossel, Very long nuclear 3He nuclear relaxation times at 4 K using cryogenic coatings, Phys. Rev. Lett. 34 (1975), 1488.

[58] G. Tastevin, P.-J. Nacher, L. Wiesenfeld, M. Leduc and F. Laloe, Obtaining polarized liquid 3He from optically oriented gas, J. Phys. (Paris) 49 (1988), 1.

[59] P.J. Nacher, G. Tastevin, M. Leduc, S.B. Crampton and F. Laloe, Spin rotation effects and spin waves in gaseous polarized 3He, J. Phys. Lett. (Paris) 45 (1984), L-441.

[60] M. Leduc, P.J. Nacher, D.S. Betts, J.M. Daniels, G. Tastevin and F. Laloe, Nuclear polarization and heat conduction changes in gaseous 3He, Europhys. Lett. 4 (1987), 59.

[61] M.E. Hayden, E. Baudin, G. Tastevin and P.-J. Nacher, NMR time-reversal as a probe of incipient turbulent spin dynamics, Phys. Rev. Lett. 99 (2007), 137602.

[62] R.S. Timsit, J.M. Daniels, E.I. Dennig, A.C.K. Kiang and A.D. May, An experiment to compressed polarized 3He gas, Bull. Am. Phys. Soc. 15 (1970), 761.

[63] J.M. Daniels, L.D. Schearer, M. Leduc and P.-J. Nacher, Polarizing 3He nuclei with neodymium La1-x Ndx Mg Al11 O19 lasers, J.O.S.A. B 4 (1987), 1133. 
[64] G. Eckert, W. Heil, M. Meyerhoff, et al., A dense polarized 3He target based on compression of optically pumped gas, Nucl. Instr. Meth. Phys. Res. A 320 (1992), 53.

[65] J. Becker, J. Bermuth, M. Ebert, et al., Interdisciplinary experiments with polarized He-3, Nuc. Instr. Meth. Phys. Res. A 402 (1998), 327.

[66] D. Rohe, P. Bartsch, D. Baumann, et al., Measurement of the neutron electric form factor $G_{\mathrm{en}}$ at $0.67(\mathrm{GeV} / \mathrm{c})^{2}$ via $3 \mathrm{He} \rightarrow\left(e \rightarrow, e^{\prime} n\right)$, Phys. Rev. Lett. 83 (1999), 4257.

[67] D.S. Hussey, D.R. Rich DR, A.S. Belov, et al., Polarized He-3 gas compression system using metastability-exchange optical pumping, Rev. Sci. Instr. 76 (2005), 053503.

[68] J. Choukeife, X. Maitre, P.J. Nacher and G. Tastevin, On-site production of hyperpolarised helium-3 gas for lung MRI, Abstracts ISSN 1524-6965 (2003), 1391.

[69] K.H. Andersen, R. Chung, V. Guillard, et al., First results from Tyrex, the new polarized-He-3 filling station at ILL, Physica B 356 (2005), 103.

[70] E.J.R. van Beek, J. Schmiedeskamp, J.M. Wild, et al., Hyperpolarized 3-helium MR imaging of the lungs: testing the concept of a central production facility, Eur. Radiol. 13 (2003), 2583.

[71] M. Batz, S. Baessler, W. Heil, et al., He-3 spin filter for neutrons, J. Res. Natl. Inst. Stand. Technol. 110 (2005), 293.

[72] A.K. Petoukhov, K.H. Andersen, D. Jullien, et al., Recent advances in polarised He-3 spin filters at the ILL, Physica B 385 (2006), 1146.

[73] J. Schmiedeskamp, W. Heil, E.W. Otten, et al., Paramagnetic relaxation of spin polarized He-3 at bare glass surfaces Part I, Eur. Phys. J. D 38 (2006), 427.

[74] A. Deninger, W. Heil, E.W. Otten, et al., Paramagnetic relaxation of spin polarized He-3 at coated glass walls Part II, Eur. Phys. J. D 38 (2006), 439.

[75] J. Schmiedeskamp, H.J. Elmers, W. Heil, et al., Relaxation of spin polarized He-3 by magnetized ferromagnetic contaminants Part III, Eur. Phys. J. D 38 (2006), 445.

[76] B. Saam, W. Happer and H. Middleton, Nuclear relaxation of 3 He in the presence of O2, Phys. Rev. A 52 (1995), 862.

[77] L. de Rochefort, A. Vignaud, X. Maitre, et al., Influence of lung filling on $T_{2}^{*}$ values in human at 1.5 T with hyperpolarised ${ }^{3}$ He, Abstracts ISSN 1545-4436 (2004), 2724.

[78] E. Durand, G. Guillot, L. Darrasse, et al., CPMG measurements and ultrafast imaging in human lungs with hyperpolarized helium-3 at low field (0.1 T), Magn. Reson. Med. 47 (2002), 75.

[79] C.P. Bidinosti, J. Choukeife, G. Tastevin, A. Vignaud and P.-J. Nacher, MRI of the lung using hyperpolarized He-3 at very low magnetic field (3 mT), Magn. Reson. Mater. Phy. 16 (2004), 255.

[80] C.P. Bidinosti, J. Choukeife, P.-J. Nacher and G. Tastevin, In-vivo NMR of hyperpolarized $3 \mathrm{He}$ in the human lung at very low magnetic fields, J. Magn. Reson. 162 (2003), 122.

[81] R.W. Mair, M.I. Hrovat, S. Patz, et al., Orientation-dependent 3He lung imaging in an open access, very-low-field human MRI system, Magn. Reson. Med. 53 (2005), 745.

[82] A.M. Oros and N.J. Shah, Hyperpolarized xenon in NMR and MRI, Phys. Med. Biol. 49 (2004), R105. 
[83] cf. http://www.phil.ens.fr. The PHIL project has been supported by the EC contract QLG1-2000-01559 running from 12/2000 to 06/2004.

[84] cf. http://www.phelinet.eu. Phelinet is an EC-funded Research and Training Network (RTN) of the 6th Framework Program (2007-2010). It involves 11 academic laboratories in 7 countries and 6 industrial partners.

[85] T. Stavngaard, L. Vejby Sogaard, J. Mortensen, et al., Hyperpolarised 3He MRI and $81^{\mathrm{m}} \mathrm{Kr}$ SPECT in chronic obstructive pulmonary disease, Eur. J. Nucl. Med. Mol. Imaging 32 (2005), 448.

[86] J.M. Wild, M.N.J. Paley, L. Kasuboski, et al., Dynamic radial projection MRI of inhaled hyperpolarized 3He gas, Magn. Reson. Med. 49 (2003), 991.

[87] E.J.R van Beek, J.M. Wild, H.U. Kauczor, et al., Functional MRI of the lung using hyperpolarized 3-helium gas, J. Magn. Reson. Imaging, 20 (2004), 540.

[88] F. Lehmann, B. Eberle, K. Markstaller, et al., Ein Auswerteprogramm zur Quantitativen Analyse von Messungen des Alveolären Sauerstoffpartialdrucks (paO2) mit der Sauerstoffsensitiven 3He-MR-Tomographie, Fortschr Roentgenstr 176 (2004), 1390.

[89] A.J. Deninger, B. Eberle, J. Bermuth, et al., Assessment of a single-acquisition imaging sequence for oxygen-sensitive 3He-MRI, Magn. Reson. Med. 47 (2002), 105114.

[90] M. Salerno, E.E. de Lange, T.A. Altes, et al., Emphysema: hyperpolarized helium 3 diffusion MR Imaging of the lungs compared with spirometric indexes - Initial experience, Radiology 222 (2002), 252.

[91] T.A. Altes, J. Mata, E.E. de Lange, J.R. Brookeman and J.P. Mugler III, Assessment of lung development using hyperpolarized helium-3 diffusion MR Imaging, J. Magn. Reson. Imaging 24 (2006), 1277.

[92] T.A. Altes, J. Mata, D.K. Froh, A. Paget-Brown, E.E. de Lange and J.P. Mugler, Abnormalities of lung structure in children with bronchopulmonary dysplasia as assessed by diffusion of hyperpolarized helium-3 MRI, Proc. Intl. Soc. Mag. Reson. Med. 14 (2006), 86.

[93] I.R. Young, Significant events in the development of MRI, J. Magn. Reson. Imaging, 19 (2004), 525.

Pierre-Jean Nacher

Laboratoire Kastler Brossel

ENS, UPMC and CNRS

24 , rue Lhomond

F-75005 Paris

France 